TITLE:

Two-dimensional finite deformations evaluated from preand post-deformation markers: Application to balanced cross sections

\author{
AUTHOR(S): \\ Yamaji, Atsushi
}

CITATION:

Yamaji, Atsushi. Two-dimensional finite deformations evaluated from pre- and postdeformation markers: Application to balanced cross sections. Journal of Structural Geology 2013

ISSUE DATE:

2013-03

URL:

http://hdl.handle.net/2433/173369

RIGHT:

(C) 2013 Elsevier Ltd.; This is not the published version. Please cite only the published version.; この論文は出版社版でありません。引用の際に は出版社版をご確認ご利用ください。 


\title{
Two-dimensional finite deformations evaluated from pre- and post-deformation markers: Application to balanced cross sections
}

\author{
Atsushi Yamaji ${ }^{\mathrm{a}, *}$ \\ ${ }^{a}$ Division of Earth and Planetary Sciences, Graduate School of Science, Kyoto University, Kyoto 606-8502, Japan
}

\begin{abstract}
A method is proposed for determining the 2D deformation gradient tensor that represents the deformations of pre- and postdeformation markers with arbitrary shapes. The deformation is not necessarily coaxial. The tensor is evaluated in a least-square sense. Therefore, the method can deal with heterogeneous deformations, and calculate their average tensor. The inverse method has the residuals that can be directly converted to the logarithmic strain needed to transform the calculated post-deformation shapes to observed ones. In addition, we propose the measure of heterogeneity for finite deformations. The method was applied to artificial and natural data from balanced cross sections.
\end{abstract}

Keywords: strain ellipse, progressive deformation, hyperbolic geometry, non-coaxiality, least-square method

\section{Introduction}

Observation of tectonic deformations allows us to test the numerical or sandbox models that predicts deformation field (e.g., McKenzie, 1979; Erslev, 1991; Allmendinger, 1998; Iwamori, 2003; Poblet and Bulnes, 2007; Henk and Nemčok, 2008; Graveleau et al., 2012). Zhang and Hynes (1995) proposed a method to determine non-coaxial 3D deformation within such a shear zone that did not have shearing in the direction across the zone.

Here, we propose a theoretical method to determine twodimensional, deformation gradient tensor, $\boldsymbol{F}$, which represents not only coaxial but also non-coaxial deformations. The tensor can always be decomposed into left-stretch tensor and orthogonal tensor, $\boldsymbol{F}=\boldsymbol{V} \boldsymbol{R}$ (Spencer, 2004). Strain ellipse or ellipsoid depicts only the left-stretch tensor, $\boldsymbol{V}$. Knowledge of strain ellipse or ellipsoid is not enough to determine $\boldsymbol{F}$, because $\boldsymbol{R}$ is remain undetermined (Davis and Titus, 2011, p. 1052). Popular strain analysis methods such as $\mathrm{R}_{\mathrm{f}} / \phi$ techniques are insufficient. Accordingly, Zhang and Hynes (1995) utilized the orientation of a shear plane and the direction of shear to solve for $\boldsymbol{F}$. We compare pre- and post-deformation shapes of markers to determine $\boldsymbol{F}$ without assuming directions of tectonic motions.

In this work, the ellipse fitting technique of Teague (1980) was used for this purpose: Mulchrone and Choudhury (2004) proved that the technique is useful for geological strain analysis. Using the technique, we can deal with the coaxial and non-coaxial deformations of markers with arbitrary shapes. It is assumed that the markers were subject to the same deformation at least approximately. Strictly, deformation field is heterogeneous, but a coarse graining approach (e.g., Lesne, 2006) allows rough but quantitative estimation of tectonic deformations (Fig. 1). We propose a measure of heterogeneity to evaluate

\footnotetext{
*Phone: +81 75753 4266; fax: +81 757534189 .

Email address: yamaji@kueps.kyoto-u.ac.jp (Atsushi Yamaji)
}

how this assumption is valid for a given data set. Assuming steady incremental deformation, Ramberg (1975) drew deformation trajectories by the numerical integration of velocity gradient tensor. We introduce a method to draw them without the integration.

Pre-deformation shapes are rarely known in nature. However, they can be inferred in balanced cross-sections (e.g., Woodward et al., 1989). The sections place quantitative constraints on the long-term tectonic deformations. We applied our method to such sections. We ignore area changes during deformation. The validity of this treatment is discussed in the final section. The areas of deformation markers in a section are used only as the weights of data in the mathematical inversion to determine the deformation gradient tensor that represent the shape changes of the markers. The numerical examples for the present method are presented using artificial and natural data sets in $\S 5$. The important symbols used in this paper is listed in Table 1.

\section{Formulation}

\subsection{Problem statement}

The deformations that affect parts of a rock body are assumed to be represented by a deformation gradient tensor, $\boldsymbol{F}$. This tensor is defined as the transformation matrix of the pre- and post-deformation position vectors,

$$
\boldsymbol{X}=\boldsymbol{F} \boldsymbol{\Xi}
$$

where $\boldsymbol{\Xi}$ and $\boldsymbol{X}$ are the vectors, respectively. $\boldsymbol{F}$ is also called position gradient tensor (e.g., Zhang and Hynes, 1995), meaning that not the absolute but relative positions are important for describing deformation. That is, the origin of the position vectors can be chosen arbitrarily.

To determine $\boldsymbol{F}$, we use the ellipses fitted to the pre- and postdeformation configurations through Teague's (1980) technique. 
Table 1: List of symbols. The upright roman subscripts, 'i' and 'f,' are used to distinguish the quantities of pre-deformation (initial) and post-deformation (final) states, respectively.

\begin{tabular}{lll}
\hline$A^{(k)}$ & Area of the $k$ th deformation marker & \\
$d_{\mathrm{H}}()$ & Hyperbolic distance & Eq. (5) \\
$\Gamma$ & Angle of elevation of $\boldsymbol{p}$ & Fig. 4 \\
$\boldsymbol{F}$ & Deformation gradient tensor & Eq. $(1)$ \\
$\boldsymbol{F}_{\mathrm{p}}$ & Deformation gradient tensor corresponding to pure shear & Eq. $(12)$ \\
$\boldsymbol{F}_{\mathrm{r}}$ & Deformation gradient tensor corresponding to rigid-body rotation & Eq. (10) \\
$H$ & Heterogeneity & Eq. $(17)$ \\
$\mathrm{H}^{2}$ & Unit hyperboloid & Eq. (2) \\
$\boldsymbol{I}$ & Identity tensor & \\
$\boldsymbol{J}$ & Minkowski tensor & Eq. (7) \\
$\mathrm{M}^{3}$ & Three-dimensional Minkowski space & \\
$n$ & Number of deformation markers & \\
$\boldsymbol{p}$ & Pole vector of a plane in $\mathrm{M}^{3}$ & Fig. 4 \\
$R$ & Aspect ratio of an ellipse & \\
$\boldsymbol{T}_{\mathrm{p}}$ & Transformation matrix in $\mathrm{M}^{3}$ corresponding to pure shear in the physical space \\
$\boldsymbol{T}_{\mathrm{r}}$ & Transformation matrix in $\mathrm{M}^{3}$ corresponding to rigid-body rotation in the physical space & Eq. (11) \\
$x_{0}, x_{1}, x_{2}$ & Rectangular Cartesian coordinates in $\mathrm{M}^{3}$ & Eq. \\
$\phi$ & Major-axis orientation of an ellipse & Fig. 2 \\
$\rho$ & Radial coordinate on $\mathrm{H}^{2}$ & \\
$\psi$ & Tangential coordinate in $\mathrm{M}^{2}$ & Eq. (3), Fig. 2 \\
$\circ$ & Lorentzian inner product & Eq. (3), Fig. 2 \\
$*$ & Lorentzian outer product & Eq. (6) \\
$\|$ & Minkowski norm & Eq. (13) \\
\hline
\end{tabular}

(a)

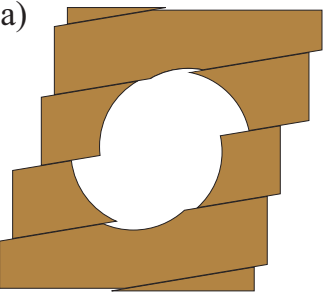

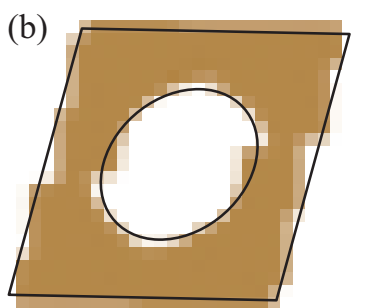

Figure 1: (a) Schematic configuration of heterogeneous deformation. (b) The deformation is approximated to a homogeneous one (solid lines) by coarse graining.

The problem we are tackling is how $\boldsymbol{F}$ is determined using the $n$ pairs of pre- and post-deformation ellipses, each of which is characterized by its aspect ratio, $R$, and major-axis orientation, $\phi$, with respect to a reference orientation in the plane where deformation is considered. We neglect area changes during deformation. This condition is written as $\operatorname{det} \boldsymbol{F}=1$, because this determinant denotes volume change (Spencer, 2004, p. 93).

Suppose that we have the $n$ pairs of ellipses that represent pre- and post-deformation markers, and let $\left(R_{\mathrm{i}}^{(k)}, \phi_{\mathrm{i}}^{(k)}\right)$ and $\left(R_{\mathrm{f}}^{(k)}, \phi_{\mathrm{f}}^{(k)}\right)$ be the paired data of the $k$ th ellipse, where the subscripts ' $\mathrm{i}$ ' and ' $\mathrm{f}$ ' distinguish the pre- and post-deformation quantities. The problem we consider, here, is how $\boldsymbol{F}$ is determined from $\left(R_{\mathrm{i}}^{(1)}, \phi_{\mathrm{i}}^{(1)}\right), \ldots\left(R_{\mathrm{i}}^{(n)}, \phi_{\mathrm{i}}^{(n)}\right)$ and $\left(R_{\mathrm{f}}^{(1)}, \phi_{\mathrm{f}}^{(1)}\right), \ldots$ $\left(R_{\mathrm{f}}^{(n)}, \phi_{\mathrm{f}}^{(n)}\right)$ with the constraint, $\operatorname{det} \boldsymbol{F}=1$.

\subsection{Necessary conditions for the parameter space of inversion}

Given the tensor, $\boldsymbol{F}$, it is possible to calculate the aspect ratio and major-axis orientation $\left(R_{\mathrm{c}}^{(k)}, \phi_{\mathrm{c}}^{(k)}\right)$ of the ellipse that is derived from $\left(R_{\mathrm{i}}^{(k)}, \phi_{\mathrm{i}}^{(k)}\right)$ (Section 3.2). Accordingly, $\boldsymbol{F}$ can be determined by mathematical inversion. That is, the optimal $\boldsymbol{F}$ is determined by minimizing the sum of the dissimilarities or distances between $\left(R_{\mathrm{c}}^{(k)}, \phi_{\mathrm{c}}^{(k)}\right)$ and $\left(R_{\mathrm{f}}^{(k)}, \phi_{\mathrm{f}}^{(k)}\right)$. The distance indicates the residual of the optimal solution. Then, how do we define the distances?

Objective methods for estimation of model parameters require optimization of a cost function, representing a measure of distance between the observations and the corresponding model predictions (e.g., Ebtehaj et al., 2010). The naive answer to the question is to use the quantity, $d=\left[\left(R_{\mathrm{f}}-R_{\mathrm{c}}\right)^{2}+\left(\phi_{\mathrm{f}}-\phi_{\mathrm{c}}\right)^{2}\right]^{\frac{1}{2}}$, as the distance for the $k$ th deformation marker. This answer implicitly use the Euclidean plane with the rectangular Cartesian coordinates, $R$ and $\phi$, with $d$ being Euclidean distance. The quantity is a bad distance measure (Yamaji, 2008, 2013), because any difference in $\phi$ does not make sense for the case of $R=1$. The significance of this difference becomes greater with increasing $R$. In addition, the ellipses with the same aspect ratio but different $\phi$ values, 0 and $180^{\circ}$, are identical, though the $\phi$ values are different.

If two ellipses are represented by the symbols, $E^{1}$ and $E^{2}$, their distance, $d\left(E^{1}, E^{2}\right)$, must satisfy the five conditions (Yamaji and Sato, 2006):

1. Non-negativity: $d\left(E^{1}, E^{2}\right) \geq 0$. 


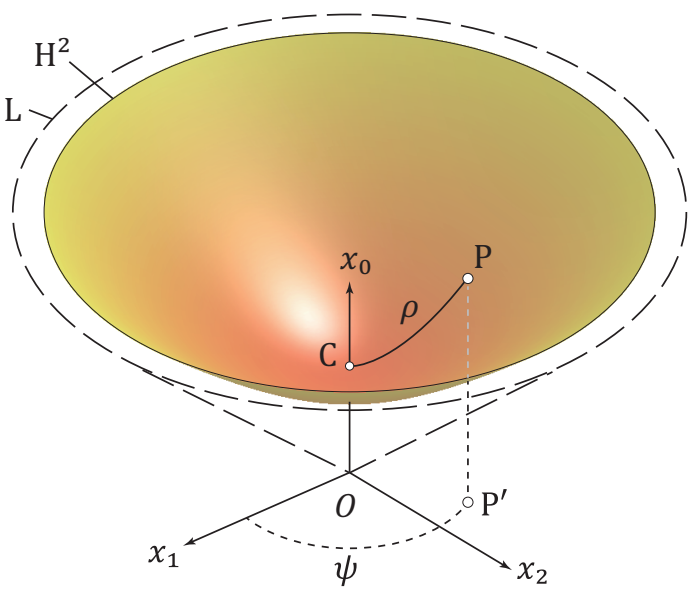

Figure 2: Schematic illustration showing the rectangular Cartesian coordinates, $O-x_{0} x_{1} x_{2}$, used in this work. $\mathrm{H}^{2}$ and $\mathrm{L}$ refer to the unit hyperboloid, $x_{0}=$ $\left(x_{1}^{2}+x_{2}^{2}+1\right)^{1 / 2}$ and the cone $x_{0}=\left(x_{1}^{2}+x_{2}^{2}\right)^{1 / 2}$, respectively. $\mathrm{H}^{2}$ has rotational symmetry about the $x_{0}$-axis, and asymptotically approaches $\mathrm{L}$ as $x_{0}$ approaches infinity. $\mathrm{C}(1,0,0)$ stands for the point at $x_{0}=1$, and C- $\rho \psi$ is the system of polar coordinates defined on $\mathrm{H}^{2}$. The point $\mathrm{P}^{\prime}$ is the orthogonal projection of the point $\mathrm{P}$ on $\mathrm{H}^{2}$ onto the $x_{1} x_{2}$-plane.

2. Identity of indiscernibles: $d\left(E^{1}, E^{2}\right)=0$ if and only if $E^{1}=E^{2}$.

3. Symmetry: $d\left(E^{1}, E^{2}\right)=d\left(E^{2}, E^{1}\right)$.

4. Triangle inequality: $d\left(E^{1}, E^{3}\right) \leq d\left(E^{1}, E^{2}\right)+d\left(E^{2}, E^{3}\right)$, where $E^{3}$ represent an ellipse.

5. Invariance: $d\left(E^{1}, E^{2}\right)$ is independent from the choice of reference orientation.

In addition, it is preferable that the distance is easily visualized and has a clear, meaningful, physical interpretation. The parameter space that was introduced by Yamaji (2008) to strain analysis fills all the demands. The distance we used in this study equals the doubled logarithmic strain needed to transform one ellipse to another by coaxial deformation. Our formulation is based on hyperbolic geometry, the non-Euclidean geometry found in the early 19th century. Concise but useful explanation on the geometry was given by Reynolds (1993).

\subsection{Hyperboloid model}

We use the unit hyperboloid, $\mathrm{H}^{2}$, expressed by the equation,

$$
x_{0}=\sqrt{x_{1}^{2}+x_{2}^{2}+1},
$$

for the parameter space to define the distances, where $x_{0}, x_{1}$ and $x_{2}$ are rectangular Cartesian coordinates (Fig. 2). This surface asymptote to the cone, $x_{0}=\sqrt{x_{1}^{2}+x_{2}^{2}}$, as $x_{0}$ approaches infinity.

We introduce the parameters,

$$
\rho=\log R \quad \text { and } \quad \psi=2 \phi,
$$

to relate the paired data $(R, \phi)$ with a point on $\mathrm{H}^{2}$, where log denotes natural logarithm. The area-preserving strain to transform a unit circle to an ellipse whose aspect ratio is $R$ has the principal strains, $R^{1 / 2}$ and $R^{-1 / 2}$. The logarithmic strain corresponding to this transformation equals $\log R^{1 / 2}=\rho / 2$. Therefore, $\rho$ equals twice the logarithmic strain. The ellipse with $(R, \phi)$ is represented by the point on $\mathrm{H}^{2}$ that is denoted by the position vector,

$$
\boldsymbol{x}=\left(\begin{array}{c}
\cosh \rho \\
\sinh \rho \cos \psi \\
\sinh \rho \sin \psi
\end{array}\right)=\frac{1}{2}\left(\begin{array}{c}
R+1 / R \\
(R-1 / R) \cos 2 \phi \\
(R-1 / R) \sin 2 \phi
\end{array}\right) .
$$

The point $\mathrm{C}(1,0,0)$ on $\mathrm{H}^{2}$ stands for an circle, i.e., $R=1$ and $\rho=0$ (Fig. 2). Long ellipses are represented by points far from C.

Fig. 3 shows the correspondence of points on $\mathrm{H}^{2}$ and the paired parameters, $(R, \phi)$, by the equal-area projection of $\mathrm{H}^{2}$ onto the $x_{1} x_{2}$-plane: $\left(x_{1}, x_{2}\right)=2 \sinh (\rho / 2)(\cos \psi, \sin \psi)$ (Reynolds, 1993). This projection is convenient for deformation analysis (Yamaji, 2013). A strain ellipse and its reciprocal strain ellipse are denoted by the points that are symmetric with respect to the center of this diagram. That is, the polar coordinates, $(\rho, \psi)$ and $(\rho, \psi+\pi)$, indicate those ellipses. This relationship is used later in the inversion to look for the optimal $\boldsymbol{F}$ for given data (Eq. 16).

We can think of $\rho$ as the distance of the point $x$ along the hyperboloid from the point $\mathrm{C}$ in Fig. 2, provided that distance between arbitrarily chosen points on $\mathrm{H}^{2}$ is denoted by

$$
d_{\mathrm{H}}(\boldsymbol{x}, \boldsymbol{y})=\cosh ^{-1}(-\boldsymbol{x} \circ \boldsymbol{y}),
$$

where $\boldsymbol{x}$ and $\boldsymbol{y}$ are the position vectors indicating the points,

$$
\boldsymbol{x} \circ \boldsymbol{y}=\boldsymbol{x}^{\mathrm{T}} \boldsymbol{J} \boldsymbol{y}=-x_{0} y_{0}+x_{1} y_{1}+x_{2} y_{2}
$$

is called Lorentzian inner product, and $\boldsymbol{J}$ is the diagonal matrix,

$$
\boldsymbol{J}=\operatorname{diag}(-1,0,0) .
$$

We call $d_{\mathrm{H}}(\boldsymbol{x}, \boldsymbol{y})$ the hyperbolic distance between the points. The introduction of this distance measure makes the threedimensional parameter space a Minkowski space (e.g., Ratcliffe, 2006). The non-Euclidean geometry with this distance measure on $\mathrm{H}^{2}$ is called hyperbolic geometry. Since $\rho$ is the hyperbolic distance from C, $\rho$ and $\psi$ can be regarded as polar coordinates lying on $\mathrm{H}^{2}$ analogous to colatitude and longitude on a sphere.

Let us introduce a few terms of hyperbolic geometry to investigate deformations. Non-zero vectors, $\boldsymbol{x}$ and $\boldsymbol{y}$, satisfying $\boldsymbol{x} \circ \boldsymbol{y}=0$ are said to be Lorentz orthogonal. It should be noted that the vectors that are Lorentz orthogonal to each other do not necessarily make a right angle. It can be seen from Eq. (6) that $\boldsymbol{x}$ with the endpoint above, on or under the cone L in Fig. 2 satisfies $\boldsymbol{x} \circ \boldsymbol{x}<0,=0$ or $>0$, respectively. The Minkowski norm of $\boldsymbol{x}$ is defined as (Ratcliffe, 2006)

$$
\|x\|=\sqrt{|x \circ x|} .
$$

It follows from Eqs. (2) and (6) that $\mathrm{H}^{2}$ is expressed as $\boldsymbol{x} \circ \boldsymbol{x}=$ -1 . This is analogous to the equation of a unit sphere, $x \cdot x=1$. 


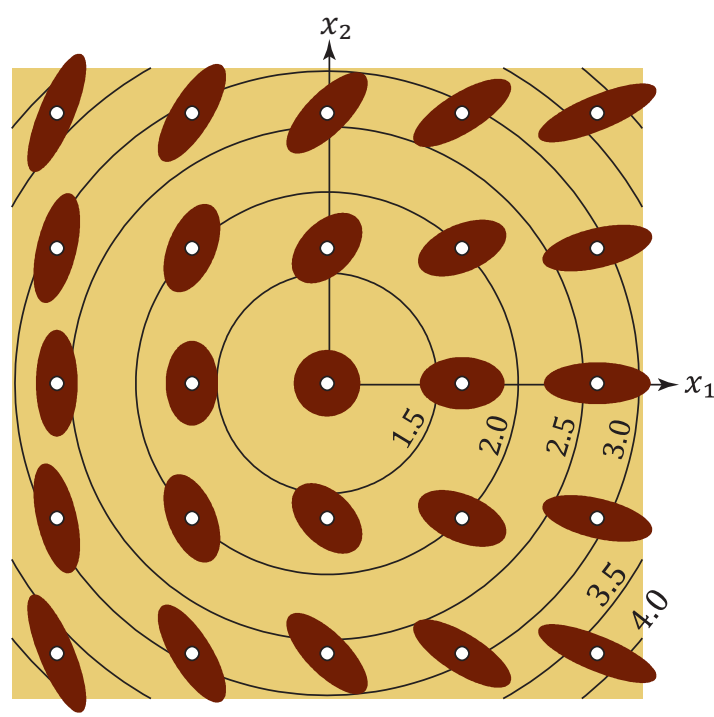

Figure 3: Equal-area projection of $\mathrm{H}^{2}$ onto the $x_{1} x_{2}$ plane, and the correspondence between ellipses in the physical spaces and points (open circles) on the plane. Concentric circles depict the contours of ellipse aspect ratio.

As this analogy suggests, spherical geometry is a useful guide for hyperbolic geometry (e.g., Vilenkin, 1968; Ratcliffe, 2006).

The geodesic between points on a sphere is the great-circle arc and is the shortest path between them, though the path is not unique if the points are antipodal to each other. The arc lies on the plane defined by the points and the center of the sphere. The geodesic between points on $\mathrm{H}^{2}$ is the unique, shortest curve that is defined by the intersection of $\mathrm{H}^{2}$ and the plane designated by the points and the origin $O$ (Fig. $4 \mathrm{a}, \mathrm{b}$ ). The length of the path equals the distance defined in Eq. (6). The vector $\boldsymbol{p}$ is called the pole of the plane, if any vector on the plane is Lorentz orthogonal to $\boldsymbol{p}$. Analogous to a great-circle and its parallels on a sphere, a geodesic has iso-distance curves on $\mathrm{H}^{2}$, which lies on the planes Lorentz orthogonal to $\boldsymbol{p}$. Each of the curves has a constant distance from the geodesic. A vector $\boldsymbol{p}$ has a geodesic on $\mathrm{H}^{2}$ if the endpoint of $\boldsymbol{p}$ is under the cone L in Fig. 2.

A circle on $\mathrm{H}^{2}$ is a closed curve, points on which have a constant hyperbolic distance from a point on $\mathrm{H}^{2}$. The latter point is the center of the circle, and is denoted by the vector $\boldsymbol{p}$. The intersection of $H^{2}$ and the plane Lorentz orthogonal to $p$ above the cone $\mathrm{L}$ is a circle (Fig. $4 \mathrm{c}$ ).

\section{Deformation in the physical space and corresponding transformation on $\mathbf{H}^{2}$}

Homogeneous deformation in the physical space is represented by the linear mapping of points on $\mathrm{H}^{2}$ that preserves hyperbolic distances between points, analogous to the preservation of great-circle distances between points during the rotation of a sphere. Such a movement or mapping that preserve distances is termed isometry (e.g., Reynolds, 1993; Ratcliffe, 2006). As a result, the spread of the points that represent elliptical deformation markers is preserved during deformation. Just like the fact that rotations in a Euclidean space is denoted by orthogonal matrix satisfying $\boldsymbol{R}^{\mathrm{T}} \boldsymbol{I} \boldsymbol{R}=\boldsymbol{I}$, the isometry is denoted by such a matrix $\boldsymbol{T}$ that satisfies $\boldsymbol{T}^{\mathrm{T}} \boldsymbol{J} \boldsymbol{T}=\boldsymbol{J}$ (Reynolds, 1993, p. 446). Then, the strain ellipse is denoted by the matrix-vector product, $\boldsymbol{T}(1,0,0)^{\mathrm{T}}$, because the position vector, $(1,0,0)^{\mathrm{T}}$, indicates a circle in the physical space (Figs. 2 and 3 ). We introduce the equations of the trajectories on $\mathrm{H}^{2}$ for incremental, steady, non-coaxial deformations in this section.

\subsection{Trajectories}

Rigid-body rotation. The simplest example of the isometry is the rotation of points on $\mathrm{H}^{2}$ about the $x_{0}$-axis by the angle $\psi_{\mathrm{r}}$, which corresponds to the rigid body rotation of ellipses by the angle, $\psi_{\mathrm{r}} / 2$, in the physical space. The factor of $1 / 2$ is the result the factor of 2 in Eq. (3). In this case, the linear mapping in the Minkowski space is denoted by the transformation matrix,

$$
\boldsymbol{T}_{\mathrm{r}}\left(\psi_{\mathrm{r}}\right)=\left(\begin{array}{ccc}
1 & 0 & 0 \\
0 & \cos \psi_{\mathrm{r}} & -\sin \psi_{\mathrm{r}} \\
0 & \sin \psi_{\mathrm{r}} & \cos \psi_{\mathrm{r}}
\end{array}\right),
$$

and the trajectories of points by this rotation are the circles centered by the point $\mathrm{C}$ on $\mathrm{H}^{2}$. The rotation around $\mathrm{C}$ represents the rotation in the physical space with the deformation gradient tensor,

$$
\boldsymbol{F}_{\mathrm{r}}\left(\psi_{\mathrm{r}} / 2\right)=\left(\begin{array}{cc}
\cos \left(\psi_{\mathrm{r}} / 2\right) & -\sin \left(\psi_{\mathrm{r}} / 2\right) \\
\sin \left(\psi_{\mathrm{r}} / 2\right) & \cos \left(\psi_{\mathrm{r}} / 2\right)
\end{array}\right) .
$$

Pure shear. The translation of points along the geodesic with the pole, $\boldsymbol{p}$, lying on the $x_{1} x_{2}$-plane represents pure shear (Fig. 4a). The orbits of points by this translation make the isodistance curves: Points on such a curve have a constant hyperbolic distance from the geodesic. The orthogonal projections of the curves onto the $x_{1} x_{2}$-plane are parallel lines (Wheeler, 1984) that make right angles with $\boldsymbol{p}$. The transformation matrix corresponding to pure shear has the form,

$$
\boldsymbol{T}_{\mathrm{p}}\left(\rho_{\mathrm{p}}, \psi_{\mathrm{p}}\right)=\boldsymbol{T}_{\mathrm{r}}\left(\psi_{\mathrm{p}}\right)\left(\begin{array}{ccc}
\cosh \rho_{\mathrm{p}} & \sinh \rho_{\mathrm{p}} & 0 \\
\sinh \rho_{\mathrm{p}} & \cosh \rho_{\mathrm{p}} & 0 \\
0 & 0 & 1
\end{array}\right) \boldsymbol{T}_{\mathrm{r}}\left(-\psi_{\mathrm{p}}\right)
$$

where the polar coordinates, $\rho_{\mathrm{p}}$ and $\psi_{\mathrm{p}}$, indicate the point on $\mathrm{H}^{2}$ corresponding to the strain ellipse (Yamaji, 2008), which is denoted by the matrix-vector product, $\boldsymbol{T}_{\mathrm{p}}\left(\rho_{\mathrm{p}}, \psi_{\mathrm{p}}\right)(1,0,0)^{\mathrm{T}}$. The pure shear has the deformation gradient tensor,

$$
\boldsymbol{F}_{\mathrm{p}}\left(\psi_{\mathrm{p}}, \psi_{\mathrm{p}}\right)=\boldsymbol{F}_{\mathrm{r}}\left(\psi_{\mathrm{p}} / 2\right) \operatorname{diag}\left(\mathrm{e}^{\rho_{\mathrm{p}} / 2}, \mathrm{e}^{-\rho_{\mathrm{p}} / 2}\right) \boldsymbol{F}_{\mathrm{r}}\left(-\psi_{\mathrm{p}} / 2\right) .
$$

Subsimple shear. General shear between pure and simple is called subsimple shear (De Paor, 1983). Such a deformation has the transformation matrix $\boldsymbol{T}=\boldsymbol{T}_{\mathrm{r}} \boldsymbol{T}_{\mathrm{p}}$ or $\boldsymbol{T}_{\mathrm{p}} \boldsymbol{T}_{\mathrm{r}}$. The corresponding trajectories on $\mathrm{H}^{2}$ lies on the planes with the pole $\boldsymbol{p}$ (Fig. 4b). The geodesic with the pole $\boldsymbol{p}$ is parameterized as $\boldsymbol{x}(t)=(\cosh t) \boldsymbol{a}+(\sinh t) \boldsymbol{p} * \boldsymbol{a}(-\infty<t<\infty)$ (Faber, 1983). where $\boldsymbol{a}$ indicates a point on the geodesic, $t$ is the hyperbolic distance of $\boldsymbol{x}$ from $\boldsymbol{a}$, and the asterisk denotes the Lorentzian outer product,

$$
\boldsymbol{x} * \boldsymbol{y}=\left(\begin{array}{c}
-\left(x_{1} y_{2}-x_{2} y_{1}\right) \\
x_{2} y_{0}-x_{0} y_{2} \\
x_{0} y_{1}-x_{1} y_{0}
\end{array}\right)
$$


(a)

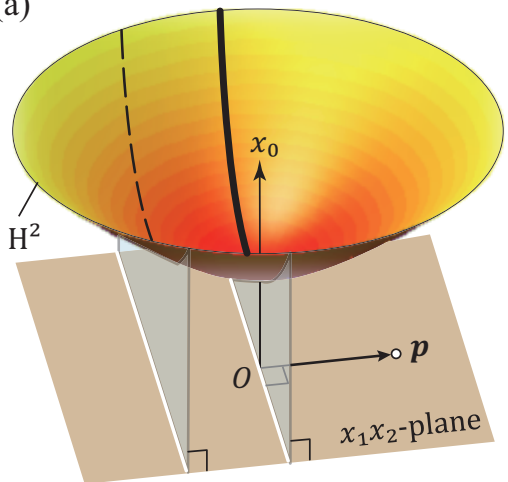

Pure shear (b)

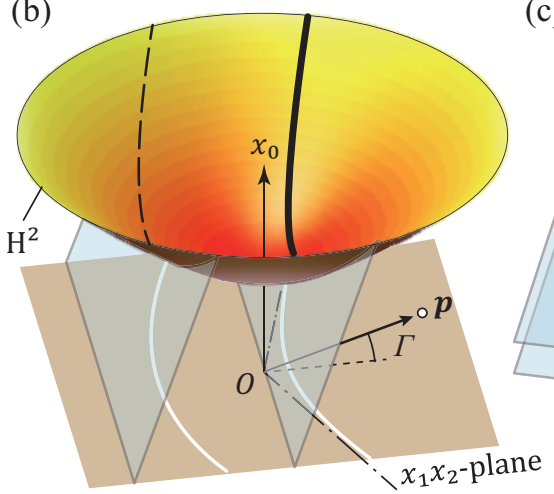

Subsimple shear

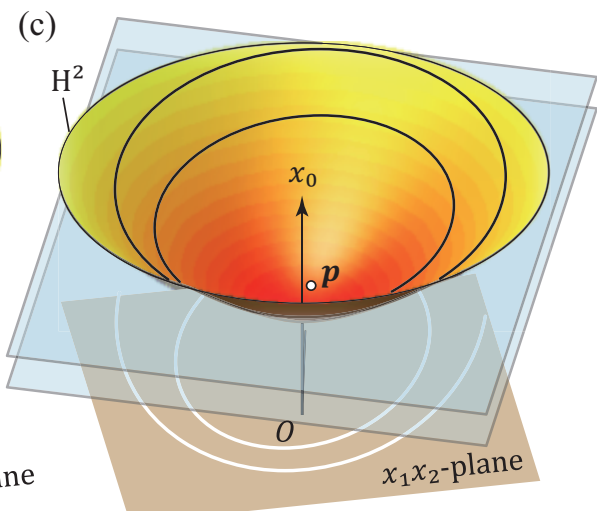

Supersimple shear

Figure 4: $(\mathrm{a}, \mathrm{b})$ Geodesic on $\mathrm{H}^{2}$ (thick line) is defined by the intersection of $\mathrm{H}^{2}$ and a plane through the origin $O$. Dashed line shows a curve that has a constant hyperbolic distance from the geodesic. The curve is the intersection of a plane parallel to the plane through the origin. The planes are Lorentz orthogonal to the pole vector $\boldsymbol{p}$. White lines show the orthogonal projections of the geodesic and its iso-distance lines onto the $x_{1} x_{2}$-plane. (c) Circles (thick lines) on $\mathrm{H}^{2}$ defined by the intersections $\mathrm{H}^{2}$ and planes. The pole of the planes, $\boldsymbol{p}$, has the endpoint on $\mathrm{H}^{2}$ that is the center of the circle. The endpoint of the vector is depicted by an open circle. The angle made by the vector and the $x_{1} x_{2}$-plane is denoted by $\Gamma$. White lines show the orthogonal projections of the circles. The curves for the two cases, $\Gamma<\pi / 4$ and $\Gamma>\pi / 4$, depict the trajectories of incremental steady deformation of sub-simple and super-simple shear, respectively, in the physical space.

The iso-distance line of this geodesic is denoted by

$$
\boldsymbol{x}(t)=(\cosh d \cosh t) \boldsymbol{a}+(\cosh d \sinh t) \boldsymbol{p} * \boldsymbol{a}+(\sinh d) \boldsymbol{p}
$$

where $\boldsymbol{a}$ indicates a point on this line, and $d=\sinh ^{-1}(\boldsymbol{p} \circ \boldsymbol{a})$ is the distance between this line and the geodesic (Nakaoka, 1993).

Simple shear. Trajectories of simple shear are parabolas, called 'horocycles,' in the Minkowski space lying on the planes with the pole on the cone L (Reynolds, 1993). The transformation matrix has the form,

$$
\boldsymbol{T}_{\mathrm{ss}}(s)=\boldsymbol{T}_{\mathrm{r}}(\psi)\left(\begin{array}{ccc}
1+s^{2} / 2 & -s^{2} / s & s \\
s^{2} / 2 & 1-s^{2} / s & s \\
s & -s & 1
\end{array}\right) \boldsymbol{T}_{\mathrm{r}}(-\psi)
$$

where $s$ and $\psi$ denote shear strain and shearing direction, respectively $(-\infty<s<\infty)$ (Reynolds, 1993). The trajectory lies on a planes that makes an angle of $45^{\circ}$ with the $x_{0}$-axis, and is parameterized as $\boldsymbol{x}(s)=\boldsymbol{a}-[(\boldsymbol{a} \cdot \boldsymbol{p}) \boldsymbol{p}] s^{2} / 2+s \boldsymbol{a} * \boldsymbol{p}$, where $\boldsymbol{a}$ indicates a point on the trajectory, and $\boldsymbol{p}$ satisfies $p_{0}=1$ and $p_{1}^{2}+p_{2}^{2}=1$ (Nakaoka, 1993).

Supersimple shear. General shear between simple shear and rigid-body rotation is termed supersimple shear (De Paor, 1983), in which the aspect ratios and major-axis orientations of deformation markers exhibit oscillatory behavior (Ramberg, 1975; Means et al., 1980). Supersimple shear has the transformation matrix $\boldsymbol{T}_{\mathrm{r}} \boldsymbol{T}_{\mathrm{p}}$ or $\boldsymbol{T}_{\mathrm{p}} \boldsymbol{T}_{\mathrm{r}}$, and is distinguished from subsimple shear by the trajectories that are circles centered by $\boldsymbol{p}$ on $\mathrm{H}^{2}$ (Fig. 4c). The circle is parameterized as

$$
\boldsymbol{x}(\vartheta)=(\cosh \varrho) \boldsymbol{a}+(\sinh \varrho \cos \vartheta) \boldsymbol{p} * \boldsymbol{a}+(\sinh \varrho \sin \vartheta) \boldsymbol{p}
$$

where $\boldsymbol{a}$ indicates a point on the circle, $\vartheta$ is the parameter in the range $0 \leq \vartheta \leq 2 \pi$ to indicates a position on the circle, and $\varrho$ is the distance of points on the circle from $\boldsymbol{p}$ (Faber, 1983; Nakaoka, 1993).

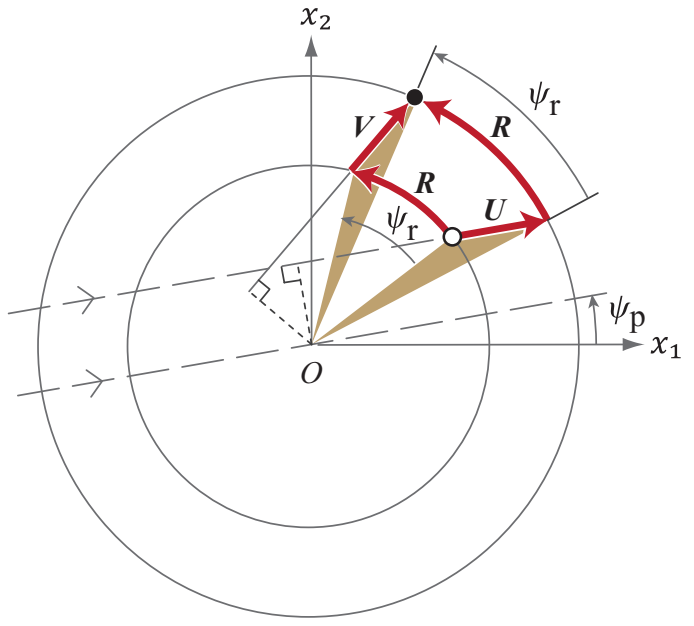

Figure 5: Orthographic projection of $\mathrm{H}^{2}$ onto the $x_{1} x_{2}$-plane for illustrating the polar decomposition, $\boldsymbol{F}=\boldsymbol{R} \boldsymbol{U}=\boldsymbol{V} \boldsymbol{R}$. Open and solid circles stand for pre- and post-deformation ellipses, respectively. The actions corresponding to $\boldsymbol{R}, \boldsymbol{U}$ and $\boldsymbol{V}$ are depicted by bold arrows. Dashed lines show the orthogonal projections of the trajectories on $\mathrm{H}^{2}$ corresponding to $\boldsymbol{U}$ : Pure shear has parallel lines as the orthogonal projections (Fig. $4 \mathrm{~b}$ ). The angle, $\psi_{\mathrm{p}}$, is twice the major-axis orientation of the maximum stretching axis of $\boldsymbol{U}$, and $\psi_{\mathrm{r}}$, is twice the angle of rigid-body rotation $\boldsymbol{R}$.

\subsection{Polar decomposition}

A deformation gradient tensor, $\boldsymbol{F}$, can always be written as $\boldsymbol{F}=\boldsymbol{R} \boldsymbol{U}=\boldsymbol{V} \boldsymbol{R}$, known as the polar decomposition, where $\boldsymbol{R}$ is the orthogonal tensor representing a rigid-body rotation, $\boldsymbol{U}$ and $\boldsymbol{V}$ are the right- and left-stretch tensors representing the pure shears whose principal axes are different from each other by the angle of $\boldsymbol{R}$ (e.g., Spencer, 2004).

We have seen that the transformation matrix, $\boldsymbol{T}_{\mathrm{r}} \boldsymbol{T}_{\mathrm{p}}$ or $\boldsymbol{T}_{\mathrm{p}} \boldsymbol{T}_{\mathrm{r}}$, in the Minkowski space denotes the non-coaxial deformations of ellipses. These matrix product are the counterparts of $\boldsymbol{R} \boldsymbol{U}$ and $\boldsymbol{V} \boldsymbol{R}$, respectively. Open and solid circles in Fig. 5 indicate 
the pre- and post-deformation ellipses. The rigid-body rotation carries points along the circles centered by $O$, and its angle of rotation, $\psi_{\mathrm{r}}$, is constant over $\mathrm{H}^{2}$ (Fig. 5). Observe the congruence of the triangles, both of which have vertices at $O$. The triangles are rotated by the angle, $\psi_{\mathrm{p}}$, about $O$, which is the result of the action of $\boldsymbol{R}$. It follows that the arrows with the labels ' $U$ ' and ' $V$ ' have the same lengths, indicating that $\boldsymbol{U}$ and $\boldsymbol{V}$ have the same amount of strain. In other words, the two deformation paths, $\boldsymbol{R}$ followed by $\boldsymbol{U}$ and $\boldsymbol{V}$ followed by $\boldsymbol{V}$, have the same result, which is meant by the polar decomposition.

\section{Determination of $F$}

\subsection{Inverse method}

In this section, we consider the methods to evaluate $\boldsymbol{F}$ from the $n$ pairs of pre- and post-deformation ellipses. Let $\boldsymbol{a}^{(1)}, \ldots$, $\boldsymbol{a}^{(n)}$ be the points on $\mathrm{H}^{2}$ standing for the pre-deformation ellipses in the physical space, and $\boldsymbol{b}^{(1)}, \ldots, \boldsymbol{b}^{(n)}$ be those for the post-deformation ones. And, let $A^{(1)}, \ldots, A^{(n)}$ be the weights of the paired data, which are normalized as $A^{(1)}+\cdots+A^{(n)}=1$. We consider the centroid (weighted mean) of the pre- and postdeformation data points,

$$
\begin{aligned}
& \overline{\boldsymbol{a}}=\frac{A^{(1)} \boldsymbol{a}^{(1)}+\cdots+A^{(n)} \boldsymbol{a}^{(n)}}{\left\|A^{(1)} \boldsymbol{a}^{(1)}+\cdots+A^{(n)} \boldsymbol{a}^{(n)}\right\|} \\
& \overline{\boldsymbol{b}}=\frac{A^{(1)} \boldsymbol{b}^{(1)}+\cdots+A^{(n)} \boldsymbol{b}^{(n)}}{\left\|A^{(1)} \boldsymbol{b}^{(1)}+\cdots+A^{(n)} \boldsymbol{b}^{(n)}\right\|},
\end{aligned}
$$

the denominators of which are the normalizing factors to place the endpoints of the position vectors, $\overline{\boldsymbol{a}}$ and $\bar{b}$, on $\mathrm{H}^{2}$. That is, they satisfy $\|\overline{\boldsymbol{a}}\|=\|\bar{b}\|=1$.

Homogeneous deformation in the physical space is represented by isometry on $\mathrm{H}^{2}$, which preserves distances between points, comparable to the movements of points on atating tectonic plate on the globe. The isometry results in the congruence of the two polygons on $\mathrm{H}^{2}$ that have the vertices at $\boldsymbol{a}^{(1)}, \ldots, \boldsymbol{a}^{(n)}$ and $\boldsymbol{b}^{(1)}, \ldots, \boldsymbol{b}^{(n)}$. In case of $n=2$, we have line segments instead of a polygons. The isometry can be decomposed into the two operations: (1) the translation of $\boldsymbol{a}^{(1)}, \ldots, \boldsymbol{a}^{(n)}$ to the points whose centroid coincides with $\bar{b}$, and (2) the rotation of those points around $\overline{\boldsymbol{b}}$ (Fig. 6a). Therefore, the transformation matrix that denotes the mapping between the polygons can be determined by searching for the translation and rotation. To do this, it is obvious that at least two pairs of pre- and post-deformation ellipses are necessary.

Data have errors, and deformation is heterogeneous to some extent. These factors lead to the distortion of the polygon. Therefore, the transformation matrix that maps $\boldsymbol{a}^{(1)}, \ldots, \boldsymbol{a}^{(n)}$ to $\boldsymbol{b}^{(1)}, \ldots, \boldsymbol{b}^{(n)}$ is determined in a least-square sense. Let $\boldsymbol{T}$ be the transformation matrix to be optimized from the data point on $\mathrm{H}^{2}$. Then, we define the residual by the hyperbolic distance, $d_{\mathrm{H}}\left(\boldsymbol{b}^{(k)}, \boldsymbol{T} \boldsymbol{a}^{(k)}\right)$. This is equal to the doubled logarithmic strain to transform ellipses represented by $\boldsymbol{b}^{(k)}$ and $\boldsymbol{T} \boldsymbol{a}^{(k)}$. The optimal transformation matrix is determined by minimizing the total squared residuals,

$$
f(\alpha, \beta, \Gamma)=\sum_{k=1}^{n} A^{(k)} d_{\mathrm{H}}\left(\boldsymbol{b}^{(k)}, \boldsymbol{T}(\alpha, \beta, \Gamma) \boldsymbol{a}^{(k)}\right)^{2},
$$
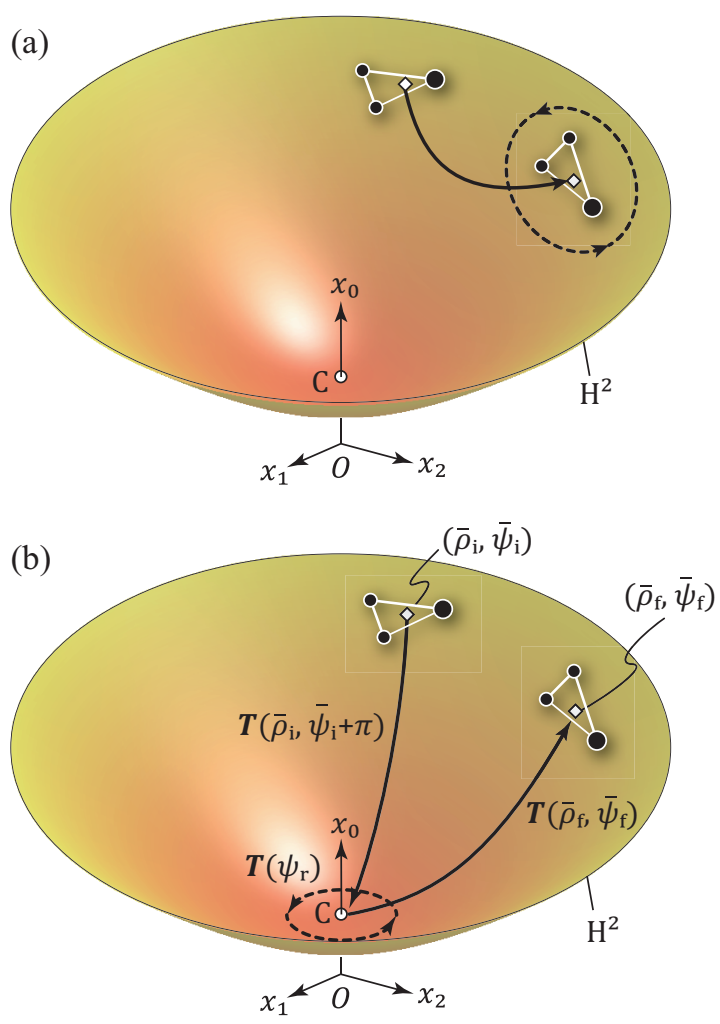

Figure 6: (a) Homogeneous deformation in the physical space is represented by the translation of points upon $\mathrm{H}^{2}$, during which hyperbolic distance between the points is preserved. The position vectors, $\boldsymbol{a}^{(i)}$ and $\boldsymbol{b}^{(i)}$, represent the preand post-deformation ellipses, respectively, by their endpoints $(i=1,2, \ldots$, $n)$. The translation can be decomposed into the two actions: the translation along a geodesic (bold line) and rotation (dotted line). (b) The translation in (a) can be decomposed also into the the three actions: (1) the translation along the geodesic from the centroid (weighted mean) of $\boldsymbol{a}^{(1)}, \ldots, \boldsymbol{a}^{(n)}$ to C, (2) rotation around $\mathrm{C}$, and (3) translation along the geodesic from $\mathrm{C}$ to the centroid of $\boldsymbol{b}^{(1)}, \ldots, \boldsymbol{b}^{(n)}$. The centroids are indicated by diamonds. The incremental action of the second step rotates points around the latter centroid.

where the transformation matrix has the form,

$$
\boldsymbol{T}(\alpha, \beta, \Gamma)=\boldsymbol{T}_{\mathrm{p}}(\alpha, \beta) \boldsymbol{T}_{\mathrm{r}}(\Gamma) \boldsymbol{T}_{\mathrm{p}}\left(\bar{\rho}_{i}, \bar{\psi}_{\mathrm{i}}+\pi\right),
$$

and $\bar{\rho}_{i}$ and $\bar{\psi}_{\mathrm{i}}$ are the polar coordinates of $\overline{\boldsymbol{a}}$. The right-hand side of this equation denotes the three operations illustrated in Fig. 6b. We minimized $F(\alpha, \beta, \Gamma)$ by the simplex method (Neider and Mead, 1965) from the starting conditions, $\alpha=\bar{\rho}_{\mathrm{f}}, \beta=\bar{\psi}_{\mathrm{f}}$ and $\Gamma=0$, where $\bar{\rho}_{\mathrm{f}}$ and $\bar{\psi}_{\mathrm{f}}$ are the polar coordinates of the point $\overline{\boldsymbol{b}}$. Once the optimal parameters, $\widehat{\alpha}, \widehat{\beta}$ and $\widehat{\Gamma}$, are obtained, the deformation gradient tensor corresponding to the optimal transformation matrix is calculated in terms of Eqs. (10) and (12):

$$
\widehat{\boldsymbol{F}}=\boldsymbol{F}_{\mathrm{p}}(\widehat{\alpha}, \widehat{\beta}) \boldsymbol{F}_{\mathrm{r}}(\widehat{\Gamma}) \boldsymbol{F}_{\mathrm{p}}\left(\bar{\rho}_{i}, \bar{\psi}_{\mathrm{i}}+\pi\right)
$$

which is the counterpart of Eq (16). Then, the optimal rightstretch tensor and orthogonal tensor, which satisfy $\widehat{\boldsymbol{F}}=\widehat{\boldsymbol{R}} \widehat{\boldsymbol{U}}$, are determined by the formulas, $\widehat{\boldsymbol{U}}^{2}=\widehat{\boldsymbol{F}}^{\mathrm{T}} \widehat{\boldsymbol{F}}$ (e.g., Spencer, 2004, p. 127) and $\widehat{\boldsymbol{R}}=\widehat{\boldsymbol{F U}}^{-1}$. 
(a)

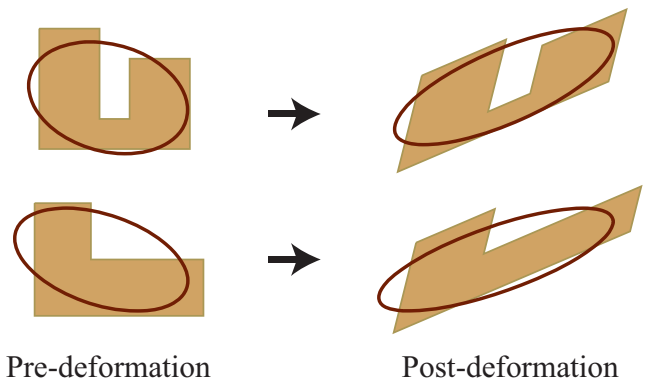

(b)

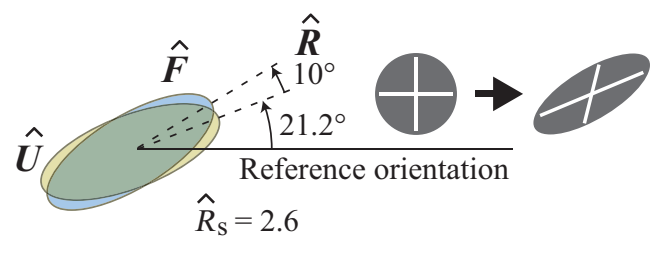

(c)

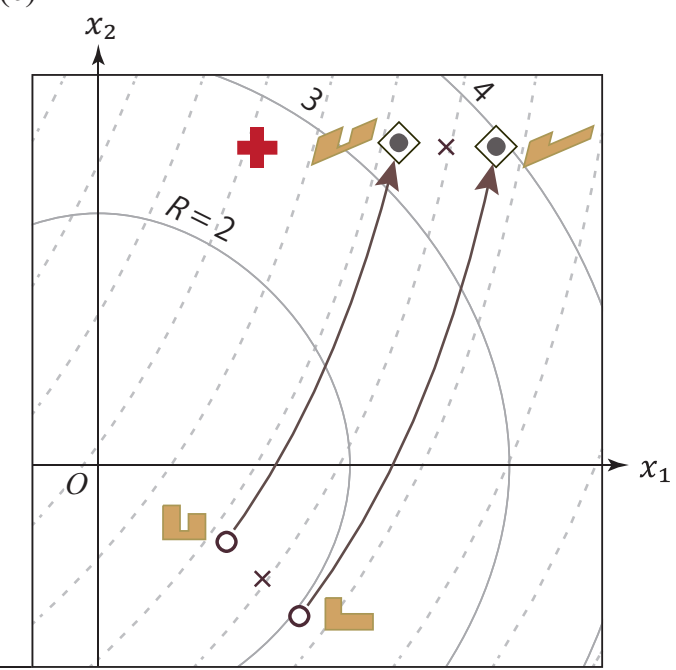

O Pre-deformation ellipse

- Post-deformation ellipse

$\diamond$ Post-deformation elllipse calculated from pre-deformation ellipses Strain ellipse

Figure 7: Example 1: Test of the present method using artificial data. (a) Pre- and post-deformation shapes and their best-fit ellipses, which were determined from the second moments of the shapes. The post-deformation shape was obtained by the deformation gradient tensor in Eq. (18). (b) Ellipses indicating the deformation tensors, $\widehat{\boldsymbol{F}}$ and $\widehat{\boldsymbol{U}}$, determined from the ellipses in (a). The hats of these symbols denote the quantities evaluated from the ellipses, the principal axes of which are depicted by dotted lines. The aspect ratio of the strain ellipse is $\widehat{R}_{\mathrm{s}}=2.6$. The rigid-body rotation of this deformation is $10^{\circ}$, corresponding to the orthogonal tensor, $\widehat{\boldsymbol{R}}$. (c) Equal-area projection of $\mathrm{H}^{2}$ showing the data points and the deformation trajectories calculated with Eq. (14).

\subsection{Heterogeneity measure}

Natural deformations are heterogeneous to some extent, though we often assume a homogeneous deformation for a set of deformation markers. Therefore, it is important to evaluate how this assumption is valid for the data set. The fact that areapreserving deformation in the physical space is represented by isometry on $\mathrm{H}^{2}$ is useful to define the heterogeneity measure. That is, if the deformation is completely homogeneous, the hyperbolic distance between $\boldsymbol{a}^{(i)}$ and $\boldsymbol{a}^{(j)}$ is equal to that between $\boldsymbol{b}^{(i)}$ and $\boldsymbol{b}^{(j)}$, where $i, j=1,2, \ldots, n$. The heterogeneity results in the elongation or shortening of the hyperbolic distance. The ratio,

$$
\epsilon^{(i j)}=\frac{d_{\mathrm{H}}\left(\boldsymbol{b}^{(i)}, \boldsymbol{b}^{(j)}\right)-d_{\mathrm{H}}\left(\boldsymbol{a}^{(i)}, \boldsymbol{a}^{(j)}\right)}{d_{\mathrm{H}}\left(\boldsymbol{a}^{(i)}, \boldsymbol{a}^{(j)}\right)},
$$

indicates the strain of the line segment between the $i$ th and $j$ th data points on $\mathrm{H}^{2}$. Accordingly, we evaluate the heterogeneity by the standard deviation of the strains between the $n$ data points,

$$
H=\sqrt{\frac{\sum_{i<j}^{n}\left[\epsilon^{(i j)}\right]^{2} A^{(i)} A^{(j)}}{\sum_{i<j}^{n} A^{(i)} A^{(j)}}} .
$$

In case of $H=0$, the same deformation affected all the deformation markers, and we have zero residuals, $d_{\mathrm{H}}\left(\boldsymbol{b}^{(k)}, \boldsymbol{T} \boldsymbol{a}^{(k)}\right)=0$ $(k=1, \ldots, n)$.

\section{Applications}

The present method was tested using artificial and natural data. Ellipses where fitted to the pre- and post-deformation shapes. Specifically, the black-and-white images of the shapes were made, and ellipses were fitted by the method of Teague (1980). This fitting was done with the software 'ImageJ' (Abramoff et al., 2004). Each image had some $1000 \times 1000$ pixels, resulting in the digitization error of the order of $1 / 1000=$ $10^{-3}$ in length.

\subsection{Example 1: Subsimple shear}

The first example (Fig. 7) demonstrates not only the feasibility of the present method but also the usability of the ellipses that are fitted to deformation markers with arbitrary shapes. Asymmetric L- and U-shaped concave polygons were used as the pre-deformation shapes (Fig. 7a). Their vertices were linearly transformed using Eq. (1) with the tensor,

$$
\boldsymbol{F}=\left(\begin{array}{ll}
1.4 & 0.2 \\
0.6 & 0.8
\end{array}\right)
$$

to derive the post-deformation shapes of the markers. These matrix components were taken from Ragan (2009, pp. 293295). This tensor has $\operatorname{det} \boldsymbol{F}=1$.

Fig. $7 \mathrm{~b}$ shows the result. We obtained the optimal tensor,

$$
\widehat{\boldsymbol{F}}=\left(\begin{array}{cc}
1.40 & 0.194 \\
0.601 & 0.798
\end{array}\right),
$$


(a)

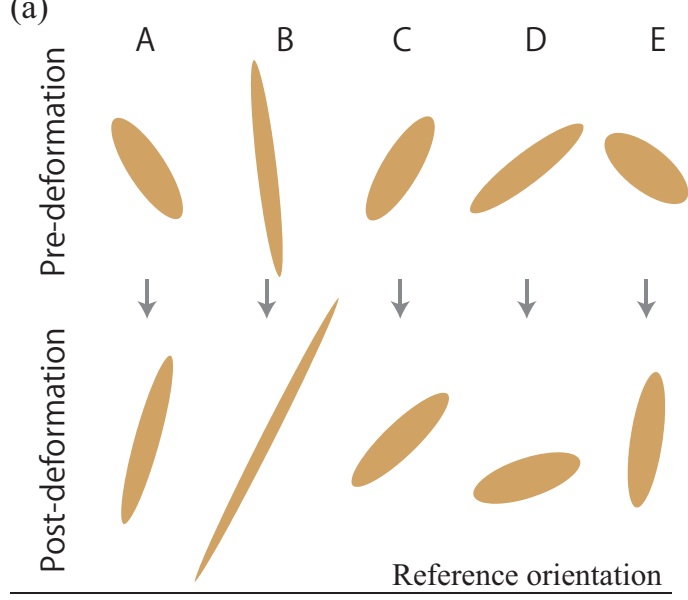

(b)

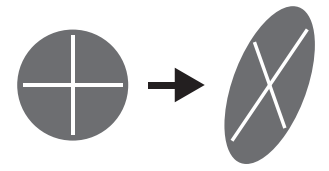

(c)

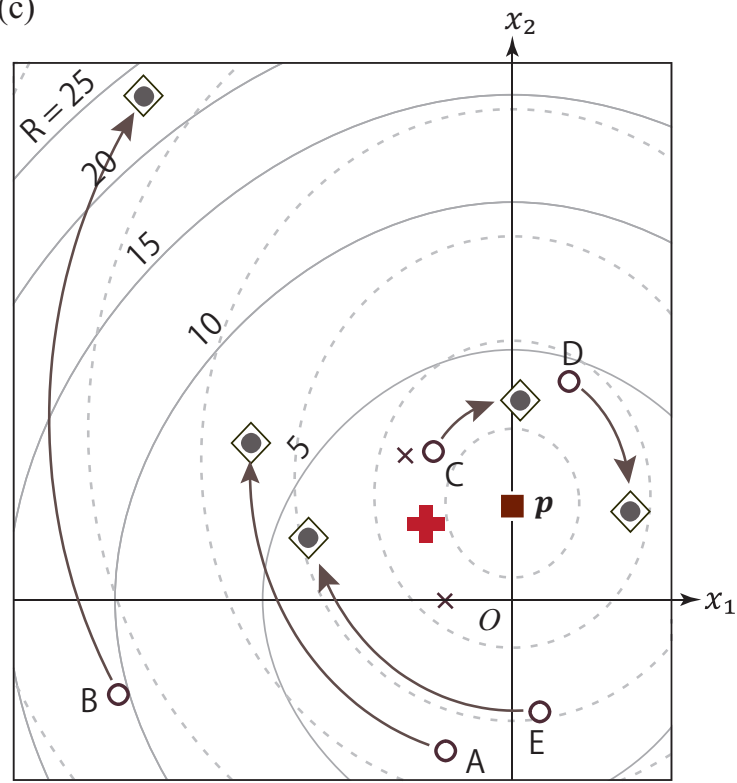

Figure 8: Example 2: Detection of supersimple shear from the five pairs of pre- and post-deformation ellipses. The pre-deformation ones were randomly generated, and the same supersimple shear was applied to them to obtain the post-deformation ones. The pairs were processed by the present method. (b) The strain ellipse corresponding to $\widehat{\boldsymbol{F}}$. (c) Equal-area projection of $\mathrm{H}^{2}$ showing the trajectories from the pre-deformation data points to the post-deformation ones calculated by Eq. (15). See Fig. 7c for legend.

which satisfactorily coincided with the assumed tensor in Eq. (18) considering the digitization error. The equal-area projection of $\mathrm{H}^{2}$ in Fig. 7c shows the trajectories calculated with Eq. (14). The post-deformation points predicted from the predeformation ones with $\widehat{\boldsymbol{F}}$ are depicted by diamonds in this figure, indicating that the predicted ones were precisely coincided with the post-deformation data points that are shown by closed circles. This coincidence demonstrates the homogeneity of the deformation. The heterogeneity measure was $H=3.4 \times 10^{-3}$, which is comparable with the digitization error of the L- and U-shapes.

\subsection{Example 2: Supersimple shear}

Artificial data for supersimple shear were made using a formula of Ramberg (1975, Eq. 72), who obtained $\boldsymbol{F}$ by integrating velocity gradient tensor. That is, the tensor we assumed was

$$
\boldsymbol{F}=\left(\begin{array}{cc}
0.4013 & 0.7131 \\
-0.7131 & 1.2247
\end{array}\right)
$$

We used randomly generated five ellipses as the predeformation markers, and post-deformation ones were generated by applying this tensor to the ellipses. Fig. 8a shows the ellipses, and Fig. 8b shows the result. We obtained the optimal tensor,

$$
\widehat{\boldsymbol{F}}=\left(\begin{array}{cc}
0.402 & 0.713 \\
-0.713 & 1.22
\end{array}\right),
$$

which had little difference from the assumed tensor in Eq. (19). Fig. 8c shows the trajectories calculated with Eq. (15). Since artificial data were used in this example, the data did not had errors except for the digitization ones of the order of $10^{-3}$. The heterogeneity measure of this example was $H=1.2 \times 10^{-3}$.

\subsection{Example 3: Natural data}

A section of an extensional duplex was used to evaluate the deformation to form the duplex (Fig. 9a). The duplex was observed in a landslide in Pliocene soft sand in central Japan that was described by Aono and Masuda (1989). Fig. 9b shows the restored configuration. The sediment had so clear lamination that we could distinguish the five layers from $\mathrm{A}$ to $\mathrm{E}$ (Fig. 9c). Four ellipses were fitted to each of the pre- and post-deformation states of the layers. That is, we had four pairs of pre- and post-deformation ellipses. For example, the first pair was composed of the layers A to E. The second one was composed of the layers A to C. Accordingly, the the best-fit ellipses had different areas. The areas were used as the weights, $A^{(1)}, \ldots, A^{(4)}$.

As a result, we obtained

$$
\widehat{\boldsymbol{F}}=\left(\begin{array}{cc}
1.19 & -0.118 \\
0.125 & 0.833
\end{array}\right)
$$

Fig. 9c shows the strain ellipse. Note that ellipses were fitted to the groups of layers with different thicknesses, but $\widehat{\boldsymbol{F}}$ approximately explains the deformations (Fig. 9c, d). It means that $\widehat{\boldsymbol{F}}$ does not depend on the width and height of the photograph from which the layers were chosen. Natural deformation only approximately satisfies our assumption that all the parts of a rock body were subject to the same deformation. Therefore, unlike the artificial data in Examples 1 and 2, the post-deformation ellipses calculated from the pre-deformation ones and $\widehat{\boldsymbol{F}}$ were slightly different from the observed post-deformation ellipses. Fig. 9e shows the small differences. Fig. 9f shows the trajectories calculated with Eq. (14). The heterogeneity measure was $H=0.25$. 


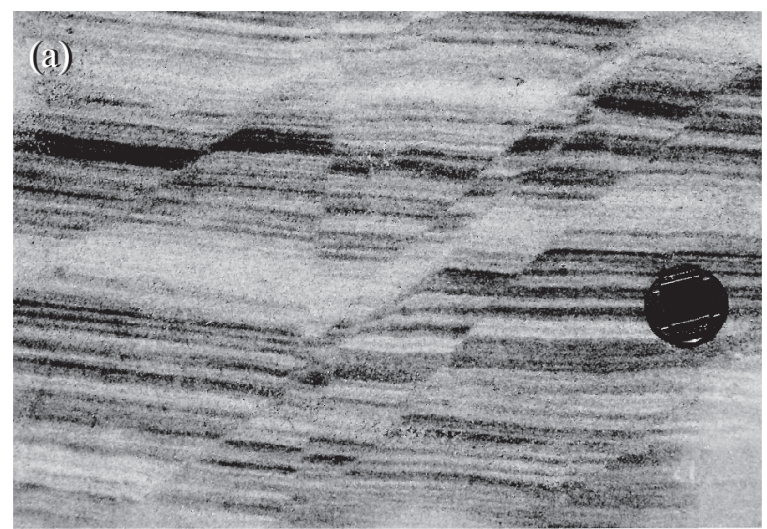

Reference orientation

(c)

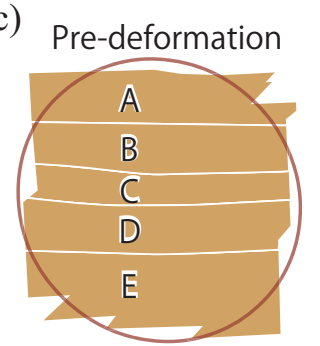

Post-deformation
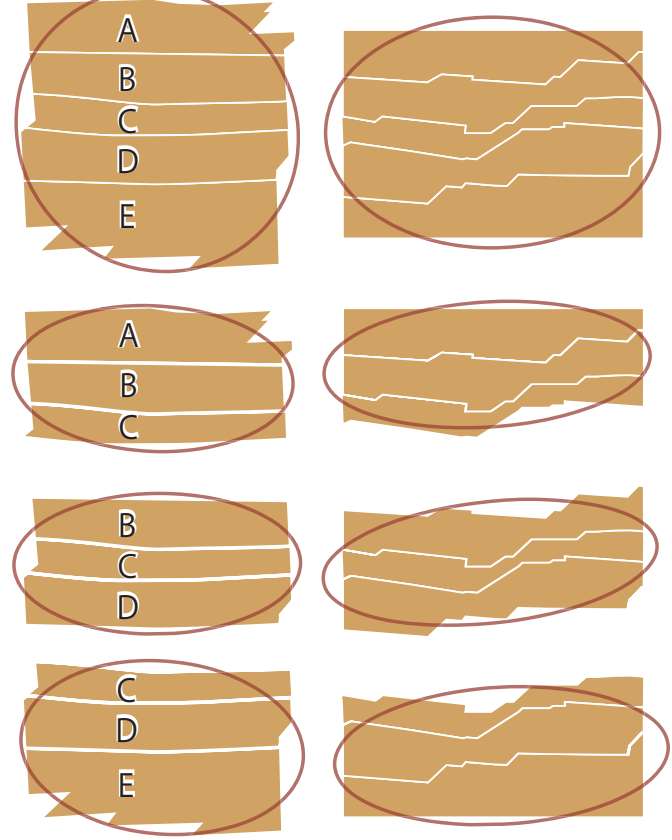

(b)

(d)

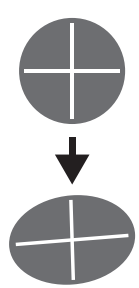

(e)

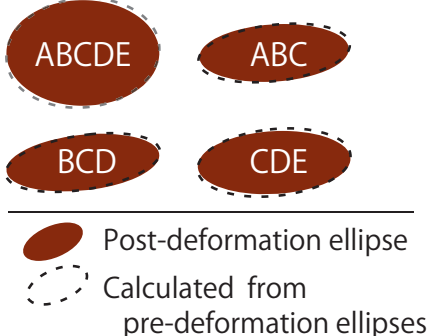

(f) $x_{2}$ pre-deformation ellipses

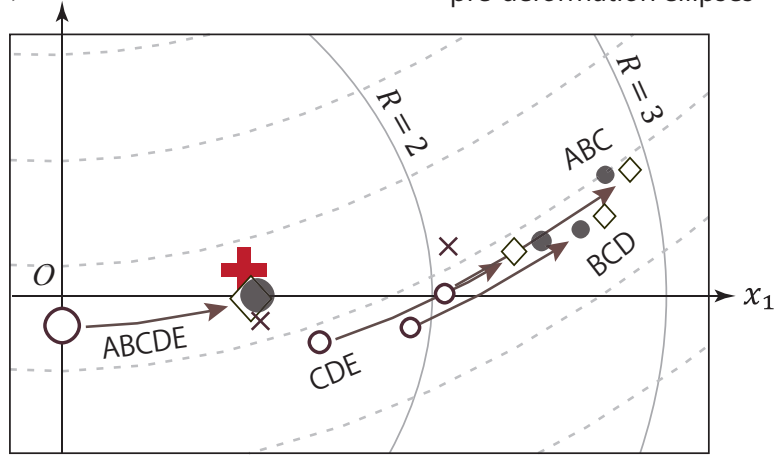

Figure 9: Example 3: (a) An outcrop scale, extensional duplex formed by submarine sliding in Upper Pliocene soft sand. The camera cap is $4 \mathrm{~cm}$ in diameter. (b) The section restored along major faults. The tilt of the strata was corrected based on the principle of original horizontality. The six faults that were used to infer the deformation from their geometric moments are highlighted. (c) Four pairs of ellipses were fitted to the pre- and post-deformation shapes of the entire and parts of the deformed sand. (d) Strain ellipse corresponding to the optimal deformation tensor $\widehat{\boldsymbol{F}}$ obtained by the present method. (e) Ellipses illustrating the residuals for the optimal deformation tensor. Dotted lines show the post-deformation ellipses calculated from the pre-deformation ones and the optimal deformation tensor. (f) Equal-area projection of $\mathrm{H}^{2}$ showing the trajectories of subsimple shear from the pre-deformation data points to the post-deformation ones. See Fig. 7c for legend. 
(a)

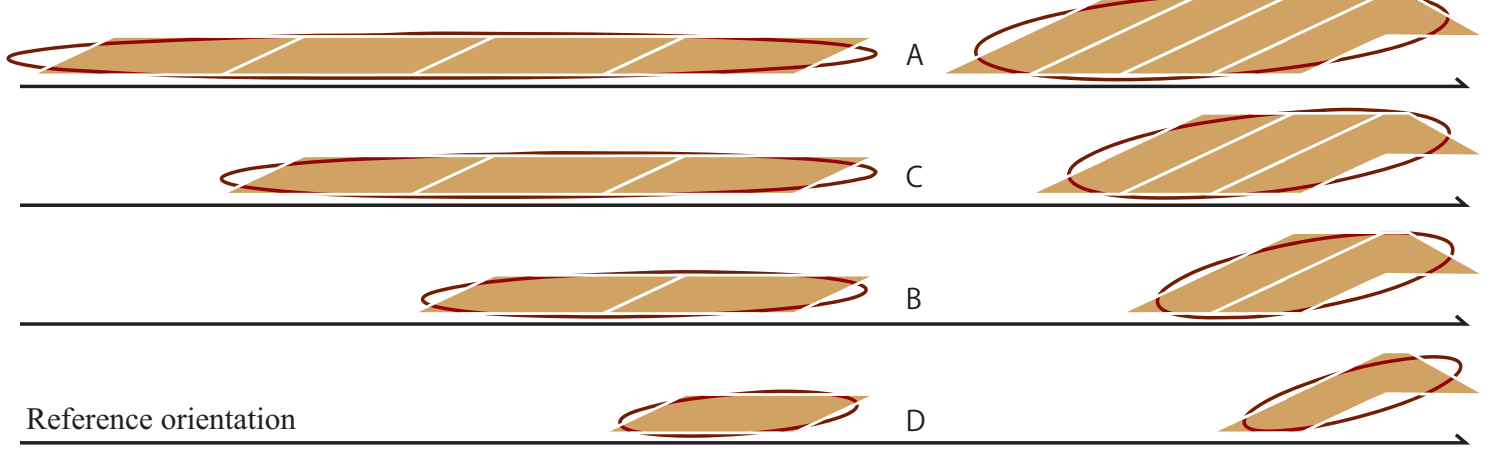

(b)

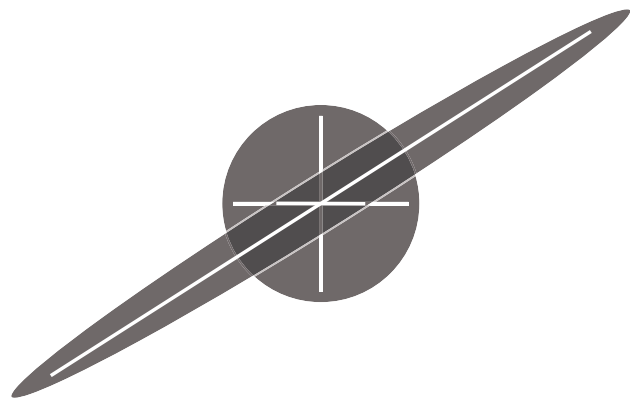

\section{(c)}

(d) $x_{2}$
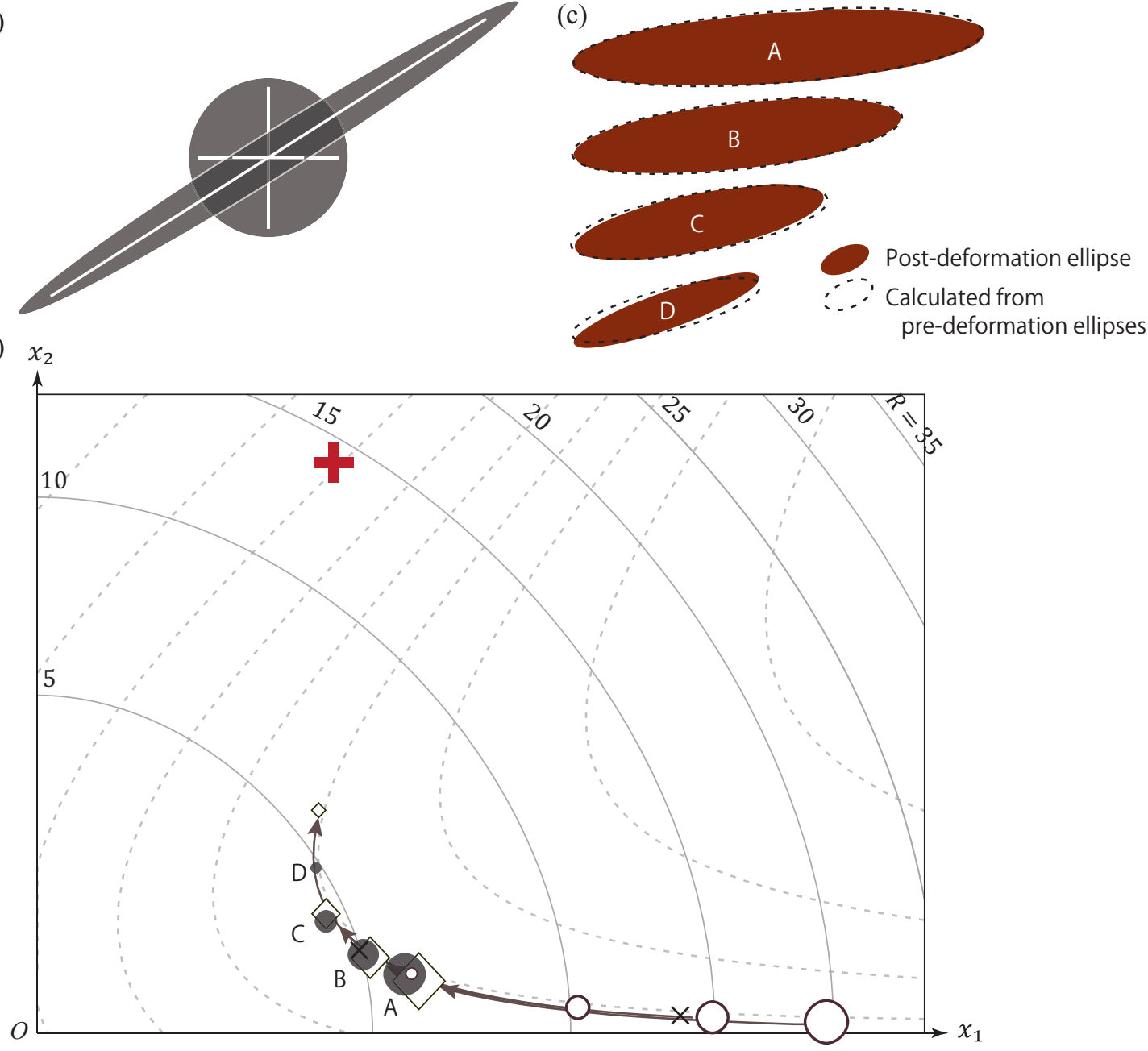

Figure 10: Example 4: Deformation evaluated from a hinterland-dipping duplex. (a) Pre- and post-deformation shapes of the duplex and their best-fit ellipses. (b) Pre-deformation unit circle and the strain ellipse deterined from the ellipses. (c) Pre-deformation and calculated ellipses. (d) Equal-area projection of $\mathrm{H}^{2}$ showing the trajectories of subsimple shear from the pre-deformation data points to the post-deformation ones. See Fig. 7c for legend. 


\subsection{Example 4: Hinterland dipping duplex}

Our method was tested with artificial data simulating a hinterland dipping duplex composed of four fault slices. Their pre- and post-deformation shapes were balanced with the kink method. Four ellipses were fitted to the entire structure and the part of the duplex (Fig. 10a).

As a result, we obtained the optimal tensor,

$$
\widehat{\boldsymbol{F}}=\left(\begin{array}{cc}
0.513 & 3.12 \\
-0.004 & 1.93
\end{array}\right)
$$

Fig. 10b shows the strain ellipse. The line of no finite rotation was more or less parallel with the sole thrust. Fig. 10c shows the fitness of the calculated post-deformation ellipses to the observed ones. The fitness was complete except for the smallest fault block D due to the effect of the anticline at the toe of the block. Formation of the anticline is a result of heterogeneous deformations, though we assume homogeneous deformation within all the blocks. The effect of the anticline increases with decreasing size of a block. Therefore, the smallest block has the largest residual. Fig. 10d shows the trajectories calculated with Eq. (14). All the points representing the pre- and post-deformation ellipses were aligned along a trajectory, indicating that the fault slices were subject to more or less the same deformation. The heterogeneity measure was $H=0.24$.

\section{Discussion}

Our formulation enjoys good properties. First, hyperbolic geometry helps geologists visualize differences in ellipses (Fig. 3 ). Second, the fitness of a best-fit model has a clear, meaningful geometric interpretation thanks to the fact that $d_{\mathrm{H}}$ indicates doubled logarithmic strain needed to transform one ellipse to another. We compare other methods that can evaluate $\boldsymbol{F}$ from pre- and post-deformation ellipses.

\subsection{Evaluation through geometric moments}

The method of Molnar (1983) estimates such deformations that were resulted from small displacements of faults by summing up the geometric moments of the faults. We applied the method to the six faults in Example 3, where the areas of faults were assumed to be equal with the length of the faults in Fig. 9a. As a result, we obtained the deformation gradient tensor,

$$
\boldsymbol{F}=\left(\begin{array}{cc}
1.18 & -0.164 \\
0.198 & 0.816
\end{array}\right),
$$

the components of which have approximate values with those determined by the present method (Eq. 20). Our method resulted in a slightly larger strain than Molnar's (Fig. 11).

In case deformation is realized by the minor displacements of planar faults, Molnar's and the present methods results in roughly the same result. However, the present one can deal with more complicated deformations involving curved faults, folds and block rotations. The hinterland dipping duplex in Fig. 10 shows such an example.

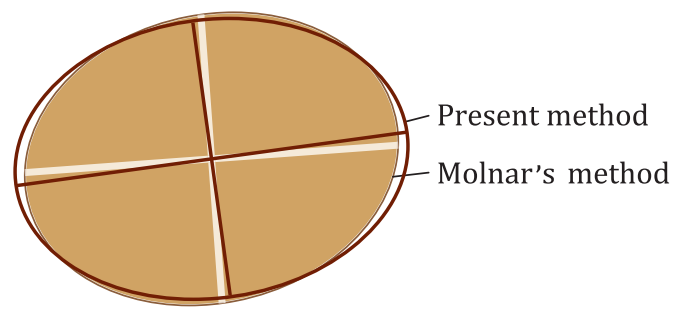

Figure 11: Strain ellipses illustrating the average deformation of the sand in Fig. 9a determined by Molnar's (1983) and the present methods. Crosses depict the principal strain axes.

\subsection{Comparison with the formulation using shape matrix}

Several authors used shape matrix of the form,

$$
\boldsymbol{N}=\left(\begin{array}{ll}
f & h \\
h & g
\end{array}\right),
$$

to deal with ellipses for strain analysis (e.g., Shimamoto and Ikeda, 1976). We use the shape matrix redefined by Wheeler (1984). That is, the equations,

$$
\begin{aligned}
& f=R^{+}+R^{-} \cos 2 \phi \\
& g=R^{+}-R^{-} \cos 2 \phi \\
& h=R^{-} \sin 2 \phi,
\end{aligned}
$$

express the relationship between shape matrix and an ellipse with the parameters, $R$ and $\phi$, where $R^{+}=(R+1 / R) / 2$ and $R^{-}=(R-1 / R) / 2$.

It is conceptually possible to evaluate $\boldsymbol{F}$ by means of the shape matrices that portray pre- and post-deformation markers. For this purpose, it is necessary to define an appropriate distance measure between shape matrices to evaluate residuals.

Utilization of matrix norm is a clue to define the distance (Yamaji and Sato, 2006). Analogous to vector length, the Frobenius norm, Frob $N=\left(N_{11}^{2}+N_{12}^{2}+N_{21}^{2}+N_{22}^{2}\right)^{1 / 2}$, indicates the 'magnitude' of a matrix (e.g., Meyer, 2000), which Choudhury and Mulchrone (2006, Eq. 3.2) employed to evaluate the difference of a shape matrix from an identity matrix. If $\boldsymbol{N}$ and $N^{\prime}$ are shape matrices, the distance between them is, for example, defined as $\operatorname{Frob}\left(\boldsymbol{N}-\boldsymbol{N}^{\prime}\right)$. It can be seen that this quantity satisfies all the five conditions of distance measure in Section 2.2.

The inversion by means of shape matrices is probably as effective as our inverse method as long as determining the optimal $\boldsymbol{F}$. However, the physical meaning of the distance measure, e.g., $\operatorname{Frob}\left(\boldsymbol{N}-\boldsymbol{N}^{\prime}\right)$, is not as clear as $d_{\mathrm{H}}$, which indicates the logarithmic strain to transform one ellipse to another. In addition, the formulation using hyperbolic geometry can consistently deal with the inversion, visualization of the differences of ellipses and quantification of the heterogeneity of deformation field.

In terms of Eq. (4), Yamaji (2013) pointed out recently that the components of shape matrix (Eq. 21) were related to a point 
on $\mathrm{H}^{2}$ :

$$
\begin{aligned}
& f=x_{0}+x_{1} \\
& g=x_{0}-x_{1} \\
& h=x_{2} .
\end{aligned}
$$

Note that the the right-hand sides of Eqs. (23) and (24) have opposite signs from the corresponding ones of Yamaji (2013, Eq. 23-25), because he used the original definition of shape matrix by Shimamoto and Ikeda (1976), which equals $\boldsymbol{N}^{-1}$. Since the transformation denoted by Eqs. (22)-(24) is linear, shape matrix has a one-to-one correspondence with a point on $\mathrm{H}^{2}$ (Yamaji, 2013). It follows from Eqs. (22)-(24) that

$$
x_{0}=(f+g) / 2, \quad x_{1}=(f-g) / 2, \quad x_{2}=h .
$$

Using these equation, the hyperbolic distance in Eq. (5) is rewritten as $d_{\mathrm{H}}=\cosh ^{-1}\left[\left(f g^{\prime}+f^{\prime} g\right) / 2-h h^{\prime}\right]$, where $f^{\prime}, g^{\prime}$ and $h^{\prime}$ are the components of $N^{\prime}$ (Eq. 21). The right-hand side of this equation denotes the 'distance' between the ellipses represented by $\boldsymbol{N}$ and $\boldsymbol{N}^{\prime}$. Therefore, if the deformation analysis using shape matrix employs the distance measure of this form, it is eventually identical with the present method. In summary, the formulation using shape matrix is not as useful as the present one because of the lack of consistent techniques for visualization and heterogeneity evaluation.

\subsection{Validity of ignoring area changes}

We show in this subsection that area changes of deformation markers can be ignored to determine deformation. A predeformation ellipse with an arbitrary area has the mathematical expression, $\boldsymbol{\Xi}^{\mathrm{T}} \boldsymbol{E}_{\mathrm{i}}^{-1} \boldsymbol{\Xi}=1$, where $\boldsymbol{\Xi}$ is the position vector of a point on the ellipse, $\boldsymbol{E}_{\mathrm{i}}$ is the symmetric $2 \times 2$-matrix representing the ellipse. The eigenvector and square-roots of the eigenvalues of $\boldsymbol{E}_{\mathrm{i}}$ indicate the principal axes and principal radii of the ellipse (e.g., Yamaji and Maeda, in press). The area of the ellipse equals $\pi \sqrt{\operatorname{det} \boldsymbol{E}_{\mathrm{i}}}$. Likewise, The post-deformation ellipse is denoted by $\boldsymbol{X}^{\mathrm{T}} \boldsymbol{E}_{\mathrm{f}}^{-1} \boldsymbol{X}=1$, where $\boldsymbol{E}_{\mathrm{f}}$ represents the post-deformation ellipse, and $\boldsymbol{X}$ the position vector of a point on the ellipse. The matrices are related with the corresponding shape matrices as

$$
\boldsymbol{N}_{\mathrm{i}}=\boldsymbol{E}_{\mathrm{i}} / \sqrt{\operatorname{det} \boldsymbol{E}_{\mathrm{i}}} \text { and } \quad \boldsymbol{N}_{\mathrm{f}}=\boldsymbol{E}_{\mathrm{f}} / \sqrt{\operatorname{det} \boldsymbol{E}_{\mathrm{f}}},
$$

where the denominators are proportional to the areas of the ellipses. Shape matrices was redefined by Wheeler (1984, Eq. A4) to satisfy these equations. Let $S_{\mathrm{i}}$ and $S_{\mathrm{f}}$ be the areas: $S_{\mathrm{i}}=\pi \sqrt{\operatorname{det} \boldsymbol{E}_{\mathrm{i}}}$ and $S_{\mathrm{f}}=\pi \sqrt{\operatorname{det} \boldsymbol{E}_{\mathrm{f}}}$. Eq. (26) say that the position vectors,

$$
\boldsymbol{y}_{\mathrm{i}}=\left(S_{\mathrm{i}} / \pi\right) \boldsymbol{x}_{\mathrm{i}} \quad \text { and } \quad \boldsymbol{y}_{\mathrm{f}}=\left(S_{\mathrm{f}} / \pi\right) \boldsymbol{x}_{\mathrm{f}} .
$$

in the Minkowski space indicate $\boldsymbol{E}_{\mathrm{i}}$ and $\boldsymbol{E}_{\mathrm{f}}$. These equations show that the vectors, $\boldsymbol{x}_{\mathrm{i}}$ and $\boldsymbol{y}_{\mathrm{i}}$, lie on a ray from the origin of the space. The same is true for $\boldsymbol{x}_{\mathrm{f}}$ and $\boldsymbol{y}_{\mathrm{f}}$. A unit circle has an area of $\pi$. Eq. (27) indicate the points in the Minkowski space that correspond to ellipses with arbitrary areas.

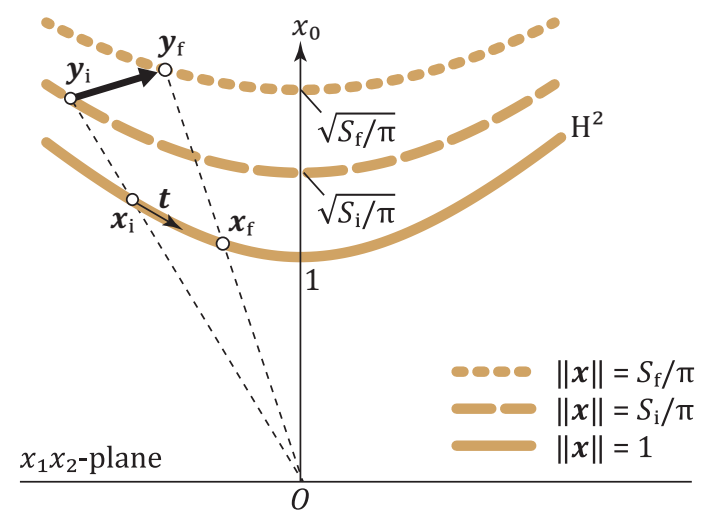

Figure 12: Hyperboloids in the Minkowski space and the linear mapping (thick arrow) between points on them. Open circles indicate the endpoints of position vectors. The vector, $\boldsymbol{t}$, is tangent to $\mathrm{H}^{2}$ at the point, $\boldsymbol{x}_{\mathrm{i}}$. Thick arrow indicates the mapping in Eq. (32). Dotted lines are rays from the origin.

The transformation between the matrices of the ellipses is written as (Yamaji and Maeda, in press, Eq. A7)

$$
\boldsymbol{E}_{\mathrm{f}}=\boldsymbol{F} \boldsymbol{E}_{\mathrm{i}} \boldsymbol{F}^{\mathrm{T}} .
$$

Combining Eqs. (26) and (28), we have

$$
\boldsymbol{N}_{\mathrm{f}}=\boldsymbol{F} \boldsymbol{N}_{\mathrm{i}} \boldsymbol{F}^{\mathrm{T}} / S,
$$

where $S=S_{\mathrm{f}} / S_{\mathrm{i}}=\operatorname{det} \boldsymbol{F}$. Combining Eqs. (22)-(25), (29) and

$$
\boldsymbol{F}=\left(\begin{array}{ll}
a & b \\
c & d
\end{array}\right),
$$

we obtain

$$
\boldsymbol{x}_{\mathrm{f}}=\boldsymbol{T} \boldsymbol{x}_{\mathrm{i}}
$$

and

$$
\boldsymbol{T}=\frac{1}{2(a d-b c)}\left(\begin{array}{ccc}
(++++) & (+-+-) & 2(a b+c d) \\
(++--) & (+--+) & 2(a b-c d) \\
2(a c+b d) & 2(a c-b d) & 2(a d+b c)
\end{array}\right)
$$

where $(++++)=+a^{2}+b^{2}+c^{2}+d^{2},(+-+-)=+a^{2}-b^{2}+$ $c^{2}-d^{2}$ and so on. It can be seen that $\operatorname{det} \boldsymbol{T}=1$ and $\boldsymbol{T}^{\mathrm{T}} \boldsymbol{J} \boldsymbol{T}=\boldsymbol{J}$, meaning that the linear mapping in Eq. (31) is isometric upon $\mathrm{H}^{2}$ (Ratcliffe, 2006). It follows from Eqs. (27) and (31) that

$$
\boldsymbol{y}_{\mathrm{f}}=(S \boldsymbol{A}) \boldsymbol{y}_{\mathrm{i}} .
$$

Owing to the factor, $S$, in this equation, the linear mapping from $\boldsymbol{y}_{\mathrm{i}}$ to $\boldsymbol{y}_{\mathrm{f}}$ is not isometric.

If the endpoint of $x_{\mathrm{i}}$ is on $\mathrm{H}^{2}$, this mapping can be decomposed into the isometry on $\mathrm{H}^{2}$ from $\boldsymbol{x}_{\mathrm{i}}$ to $\boldsymbol{x}_{\mathrm{f}}$ and the movement along the line from $\boldsymbol{x}_{\mathrm{f}}$ to $\boldsymbol{y}_{\mathrm{f}}$ (Fig. 12). These two movements represent area-preserving shape change and area change without shape change, respectively. In this case, we have $\left\|\boldsymbol{y}_{\mathrm{f}}\right\|=S$, $S_{\mathrm{i}}=\pi$ and $\left\|\boldsymbol{x}_{\mathrm{f}}\right\|=1$. Therefore the point indicated by $\boldsymbol{y}_{\mathrm{f}}$ exists on the hyperboloid, $\left\|\boldsymbol{y}_{\mathrm{f}}\right\|=S$. Let $\boldsymbol{t}$ be a vector tangent to the orbit from $\boldsymbol{x}_{\mathrm{i}}$ to $\boldsymbol{x}_{\mathrm{f}}$ at the point indicated by $\boldsymbol{x}_{\mathrm{i}}$. The tangent vector satisfies $\boldsymbol{t} \circ \boldsymbol{x}_{\mathrm{i}}=0$ (Ratcliffe, 2006, p. 73), meaning that $\boldsymbol{t}$ and 
$\boldsymbol{x}_{\mathrm{i}}$ are Lorentz orthogonal to each other. If deformation in the physical space is very small, the endpoints of the vectors, $\boldsymbol{x}_{\mathrm{i}}, \boldsymbol{x}_{\mathrm{f}}$, $\boldsymbol{y}_{\mathrm{i}}$ and $\boldsymbol{y}_{\mathrm{f}}$ make a parallelogram. Then, the vectors, $\left(\boldsymbol{x}_{\mathrm{f}}-\boldsymbol{x}_{\mathrm{i}}\right)$ and $\left(\boldsymbol{y}_{\mathrm{f}}-\boldsymbol{x}_{\mathrm{f}}\right)$, are Lorentz orthogonal to each other. This means that area-preserving shape change and shape change without area change in the physical space are independent from each other. In other word, aspect ratio, major-axis orientation and size are independent information of an ellipse. Therefore, we can ignore the area changes to determine deformation gradient tensor with the assumption, $\operatorname{det} \boldsymbol{F}=1$, at the cost of the indeterminacy of area changes.

\subsection{Counterpart of kinematic vorticity number}

Fig. 4 suggests that the angle of elevation of $\boldsymbol{p}$ has something to do with kinematic vorticity, which indicates the ratio of the effects of $\boldsymbol{R}$ and $\boldsymbol{V}$. It was found in this study that the angle, $\Gamma$, is related to kinematic vorticity number, $W_{\mathrm{k}}$ through the equation,

$$
W_{\mathrm{k}}=\tan \Gamma,
$$

for steady, incremental deformations with $\operatorname{det} \boldsymbol{F}=1$. This is shown as follows.

Mohr circle of finite deformation is related with $W_{\mathrm{k}}$ through the equation, $W_{\mathrm{k}}=q / r$, where $r$ is radius the circle, and $q$ is the distance between center of the circle and the abscissa (Passchier, 1990, Eq. 7). Given the components of deformation gradient tensor (Eq. 30), the corresponding Mohr circle is designated by the diameter spanned by the points at $(d, b)$ and $(a,-c)$ (Means, 1982). Therefore, we have $r=\sqrt{(a-d)^{2}+(b+c)^{2}} / 2$, $q=|(b-c) / 2|$, and

$$
W_{\mathrm{k}}=\frac{q}{r}=\frac{|b-c|}{\sqrt{(a-d)^{2}+(b+c)^{2}}} .
$$

On the other hand, $\boldsymbol{T}$ is the transformation matrix of the linear mapping in Eq. (31). Note the analogy between the equations, $\boldsymbol{T}^{\mathrm{T}} \boldsymbol{J} \boldsymbol{T}=\boldsymbol{J}$ and $\boldsymbol{R}^{\mathrm{T}} \boldsymbol{I} \boldsymbol{R}=\boldsymbol{I}$, the latter of which denotes rotation in the physical space. As the analogy suggests, the mapping is known as hyperbolic rotation in the Minkowski space. It means that the mapping has a 'rotation axis,' which is identical with $\boldsymbol{p}$. The pole is not moved by the mapping, resulting in the fact that $\boldsymbol{p}$ is parallel to the eigenvector of $\boldsymbol{T}$ corresponding to its eigenvalue 1. A simple calculation shows that this eigenvector has the components, $(b-c, b+c,-(a-d))^{\mathrm{T}}$. These vector components show that $\tan \Gamma$ equals the right-hand side of Eq. (34), thereby Eq. (33) being proved. It should be noted that the number is defined for instantaneous or infinitesimal deformation.

\subsection{Supplementary properties of the formulation using $H^{2}$}

Can the present formulation be adapted to three-dimensional deformation? The argument on this issue is beyond the scope of this paper, but we would like to refer to a trend of plasticity theory utilizing a higher-dimensional Minkowski space to deal with three-dimensional stress-strain relationship. That is, the series of works by Hong and Liu use equivalent strain to define the $x_{0}$-axis of six-dimensional Minkowski space (e.g., Hong and Liu, 1999, 2001), where the remaining five-dimensional subspace is unexpectedly equivalent with the parameter space recently employed in the analyses of three-dimensional paleostresses (Sato and Yamaji, 2006; Yamaji and Sato, 2006; Otsubo et al., 2008; Yamaji et al., 2010) and three-dimensional shape fabric (Yamaji et al., 2007). The theory is a clue to extend the present method to three-dimensions.

Is the error estimation of $\boldsymbol{F}$ possible? To answer this problem, distance must be defined between deformation gradient tensors. It poses a challenge: the amount of subsimple shear goes to infinity, but that of supersimple shear shows an oscillatory behavior. In the latter case, initial configurations of deformation markers appear many times during steady, incremental deformations. A single distance measure should evaluate the amount of deformation consistently for sub- and supersimple shear and simple shear. The problem is unanswered.

\section{Acknowledgments}

The author would like to thank Joshua R. Davis and Kieran Mulchrone for helpful suggestions which greatly improved the manuscript. This work was financially supported by JSPS (22340151).

\section{Appendix A. Supplementary materials}

This article has the following supplementary materials.

- Eighteen MATLAB M-files for the present inverse method. The file 'main0.m' is the main program.

- File 'readme.txt' containing the explanations for the program.

- Files 'LU.tif' and 'LU.xls' containing the sample data shown in Fig. 7.

\section{References}

Abramoff, M.D., Magalhaes, P.J., Ram, S.J., 2004. Image Processing with ImageJ. Biophotonics International 11. 36-42.

Allmendinger, 1R.W., 1998. Inverse and forward numerical modeling of trishear fault-propagation folds. Tectonics 17, 640-656.

Aono, H., Masuda, F., 1989. Synsedimentary conjugate faults in the Pleistocene tidal deposits at Ushibori, Ibaraki, Japan. Taira, A., Masuda, F., eds., Sedimentary Facies in the Active Margin, Terrapub, Tokyo, pp. 143-149.

Bobyarchick, A.R., 1986. The eigenvalues of steady flow in Mohr space. Tectonophysics 122, 35-51.

Choudhury, K.R. Mulchrone K.F., 2006. A comparative error analysis of manual versus automated methods of data acquisition for algebraic strain estimation. Tectonophysics 421, 209-230.

De Paor, D., 1983. Orthographic analysis of geological structures-I. Deformation theory. Journal of Structural Geology 5, 255-277.

Ebtehaj, M., Moradkhani, H., Gupta, H.V., 2010. Improving robustness of hydrologic parameter estimation by the use of moving block bootstrap resampling. Water Resources Research 46, W07515, doi:10.1029/2009WR007981.

Erslev, E.A., 1991. Trishear fault-propagation folding. Geology 19, 617-620.

Faber, R.L., 1983. Foundations of Euclidean and Non-Euclidean Geometry. Dekker, New York, 329pp.

Henk, A., Nemčok, M., 2008. Stress and fracture prediction in inverted halfgraben structures. Journal of Structural Geology 30, 81-97. 
Ghosh, S.K., 1987. Measure of non-coaxiality. Journal of Structural Geology 9, 111-113.

Graveleau, F., Malavieille, J., Dominguez, S., 2012. Experimental modelling of orogenic wedges: A review. Tectonophysics 538-540, 1-66.

Grote, K.-H., Antonsson, E.K. (Eds.), 2009. Springer Handbook of Mechanical Engineering. Springer, Berlin.

Hong, H.-K., Liu, C-S., 1999. Lorentz group $\mathrm{SO}_{0}(5,1)$ for perfect elastoplasticity with large deformation and a consistency numerical scheme. International Journal of Non-Linear Mechanics 34, 1113-1130.

Hong, H.-K., Liu, C-S., 2001. Some physical models with Minkowski spacetime structure and Lorentz group symmetry. International Journal of NonLinear Mechanics 36, 1075-1084.

Iwamori, H., 2003. Viscous flow and deformation of regional metamorphic belts at convergent plate boundaries. Journal of Geophysical Research 108 , 2321, doi:10.1029/2002JB001808

Lesne, A., 2006. Multiscale approaches. In, Francoise, J.-P., Naber, G.L. and Tsun, T.S., Encyclopedia of Mathematical Physics, 465-482, Elsevier

McKenzie, D., 1979. Finite deformation during fluid flow. Geophysial Journal of the Royal Society 58, 689-715.

Means, W.D,, 1982. An unfamiliar Mohr circle construction for finite strain. Tectonophysics 89, T1-T6.

Means, W.D., Hobbs, B.E., Lister, G.S., Williams, P.F., 1980. Vorticity and non-coaxiality in progressive deformations. Journal of Structural Geology 2, 371-378.

Nelder, J.A., Mead, R., 1965. A simplex method for function minimization. Computer Journal 7, 308-313.

Meyer, C.D., 2000. Matrix Analysis and Applied Linear Algebra. SIAM, Philadelphia.

Molnar, P., 1983. Average regional strain due to slip on numerous faults of different orientations. Journal of Geophysical Research 88, 6430-6432.

Mulchrone K.F., Choudhury, K.R. 2004. Fitting an ellipse to an arbitrary shape: implications for strain analysis. Journal of Structural Geology 26, 143-153.

Nakaoka, M., 1993. Introduction to Hyperbolic Geometry: Application of Linear Algebra (in Japanese). Saiensu-sha, Tokyo.

Otsubo, M., Yamaji, A., Kubo, A., 2008. Determination of stresses from heterogeneous focal mechanism data: An adaptation of the multiple inverse method. Tectonophysics 457, 150-160.

Passchier, C.W., 1988. The use of Mohr circles to describe non-coaxial progressive deformation. Tectonophysics 149, 323-338.

Passchier, C.W., 1990. Reconstruction of deformation and flow parameters from deformed veins sets. Tectonophysics 180, 185-199.

Passchier, C.W., Trouw, R.A.J., 2005. Microtectonics, 2nd Edition. Springer, Berlin.

Poblet, J., Bulnes, M., 2007. Predicting strain using forward modelling of restored cross-sections: Application to rollover anticlines over listric normal faults. Journal of Structural Geology 29, 1960-1970.

Provost, A., Buisson, C., Merle, O., 2004. From progressive to finite deformation and back. Journal of Geophysical Research 109, B02405, doi:10.109/2001JB001734.

Ragan, D.M., 2009. Structural Geology: An Introduction to Geometrical Techniques, Fourth Edition. Cambridge University Press, Cambridge.

Ramberg, H., 1975. Particle paths, displacement and progressive strain applicable to rocks. Tectonophysics $28,1-37$.

Ratcliffe, J,G,, 2006. Foundations of Hyperbolic Manifolds, Second Edition. Springer Science and Business Media, New York, 779 pp.

Reynolds, W.F., 1993. Hyperbolic Geometry on a Hyperboloid. American Mathematical Monthly 100, 442-455.

Sato, K., Yamaji, A., 2006. Embedding stress difference in parameter space for stress tensor inversion. Journal of Structural Geology, 28, 957-971.

Shimamoto, T., Ikeda, Y., 1976. A simple algebraic method for strain estimation from deformed ellipsoidal objects: 1 . Basic theory. Tectonophysics 36,315 337.

Spencer, A.J.M., 2004. Continuum Mechanics. Dover Publication, Mineola.

Teagure, M.R., 1980. Image analysis via the general theory of moments. Journal of Optical Society of America 70, 920-930.

Davis, J.R., Titus, S.J., 2011. Homogeneous steady deformation: A review of computational techniques. Journal of Structural Geology 33, 1046-1062.

Torsvik, T.H., Müller, R.D., Van der Voo, R., Steinberger, B., Gaina, C., 2008. Global plate motion frames: Toward a unified model. Reviews of Geophysics 46, RG3004, doi:10.1029/2007RG000227.

Truesdell, C., 1953. Two measures of vorticity. Journal of Rotational Mechani- cal Analysis 2, 173-217

Vilenkin, N.J., 1968. Special Functions and the Theory of Group Representation. In: Translations of Mathematical Monographs 22. American Mathematical Society.

Wheeler, J., 1984. A new plot to display the strain of elliptical markers. Journal of Structural Geology 6, 417-423

Wheeler, J., 1986. Average properties of ellipsoidal fabrics: Implications for two- and three-dimensional methods of strain analysis. Journal of Structural Geology 126, 259-270.

Woodward, N.B., Boyer, S.E., Suppe, J., 1989. Balanced Geological CrossSections: An Essential Technique in Geological Research and Exploration. American Geological Union, Washington D.C.

Yamaji, A., 2008. Theories of strain analysis from shape fabrics: A perspective using hyperbolic geometry. Journal of Structural Geology 30, 1451-1465.

Yamaji, A., 2013. Comparison of methods of algebraic strain estimation from $\mathrm{R}_{\mathrm{f}} / \phi$ data: A unified theory of $2 \mathrm{D}$ strain analysis. Journal of Structural Geology, doi:10.1016/j.jsg.2013.01.011.

Yamaji, A., Maeda, H., in press. Determination of 2D strain from a fragmented single ammonoid. Island Arc, doi:10.1111/iar.12006.

Yamaji, A., Sato, K., 2006. Distances for the solutions of stress tensor inversion in relation to misfit angles that accompany the solutions. Geophysical Journal International, 167, 913-942.

Yamaji, A., Sato, K., Tonai, S., 2010. Stochastic modeling for the stress inversion of vein orientations: Paleostress analysis of Pliocene epithermal veins in southwestern Kyushu, Japan. Journal of Structural Geology, 32, 11371146.

Yamaji, A., Yokokawa, M., Sato, K., 2007. Analysis of clustering in threedimensional grain fabric. Geosphere 3, 108-118.

Zhang, G., Hynes, A., 1995. Determination of position-gradient tensor from strain measurements and its implication for the displacement across a shear zone. Journal of Structural Geology 17, 1587-1599. 